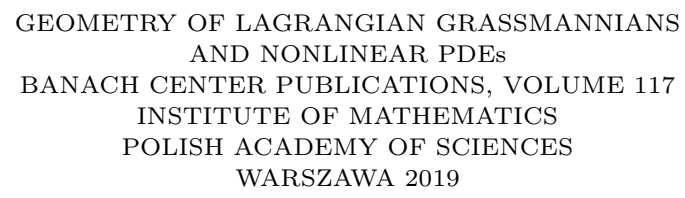

\title{
GEOMETRY OF LAGRANGIAN GRASSMANNIANS AND NONLINEAR PDEs
}

\author{
JAN GUTT \\ Center for Theoretical Physics of the Polish Academy of Sciences \\ Al. Lotników 32/46, 02-668 Warsaw, Poland \\ E-mail: jan.gutt@gmail.com
}

GIANNI MANNO

Dipartimento di Scienze Matematiche "G. L. Lagrange", Politecnico di Torino

Corso Duca degli Abruzzi, 24, 10129 Torino, Italy

E-mail: giovanni.manno@polito.it

\section{GIOVANNI MORENO}

Department of Mathematical Methods in Physics, University of Warsaw

ul. Pasteura 5, 02-093 Warszawa, Poland

E-mail: giovanni.moreno@fuw.edu.pl

\begin{abstract}
This paper contains a thorough introduction to the basic geometric properties of the manifold of Lagrangian subspaces of a linear symplectic space, known as the Lagrangian Grassmannian. It also reviews the important relationship between hypersurfaces in the Lagrangian Grassmannian and second-order PDEs. Equipped with a comprehensive bibliography, this paper has been especially designed as an opening contribution for the proceedings volume of the homonymous workshop held in Warsaw, September 5-9, 2016, and organised by the authors.
\end{abstract}

2010 Mathematics Subject Classification: 35A30, 53C30, 53D10.

Key words and phrases: Nonlinear PDEs, exterior differential systems, contact geometry, Lagrangian Grassmannians, projective duality, integrability.

Research founded by the Polish National Science Centre grant under the contract number 2016/22/M/ST1/00542.

The paper is in final form and no version of it will be published elsewhere. 


\section{Contents}

Introduction . . . . . . . . . . . . . . . . . . . . . . . . . 10

1. Geometry of the (real and complex) Lagrangian Grassmannian . . . . . . . . . . . . 12

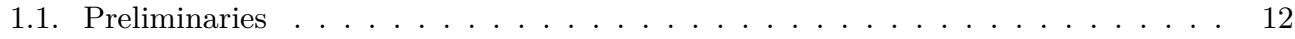

1.2. Definition of the Lagrangian Grassmannian . . . . . . . . . . . . . . . . . 12

1.3. Coordinate-free definition of the Lagrangian Grassmannian . . . . . . . . . . . . 14

1.4. The Plücker embedding . . . . . . . . . . . . . . . . . . . . . . . . 14

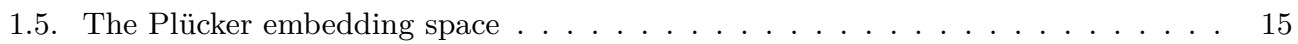

1.6. The Plücker relations . . . . . . . . . . . . . . . . . . . . . . . . . 16

1.7. The dual variety $\ldots \ldots \ldots \ldots \ldots \ldots \ldots \ldots$

1.8. Natural group actions on $\operatorname{LGr}\left(V \oplus V^{*}\right) \ldots \ldots \ldots \ldots$

1.9. The homogeneous structure of $\operatorname{LGr}\left(V \oplus V^{*}\right) \ldots \ldots \ldots \ldots$. . . . . . . . . . 21

1.10. The tautological and the tangent bundle of $\operatorname{LGr}(n, 2 n) \ldots \ldots$. . . . . . . . . . . . . . . . . . . . . . . . . . . .

1.11. The second-order frame bundle . . . . . . . . . . . . . . . . . . . . . . 24

1.12. Representation theory of $\mathrm{Sp}_{2 n}$ and its subgroup $\mathrm{GL}_{n} \ldots \ldots \ldots \ldots$. . . . . . 24

1.13. The tautological line bundle and $r$-th degree hypersurface sections . . . . . . . 25

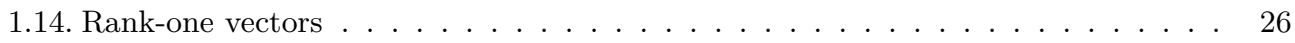

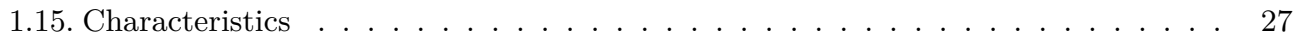

1.16. The Lagrangian Chow transform . . . . . . . . . . . . . . . . . . . . . . . . . . . . . . . . . . . .

1.17. A few remarks on $\operatorname{LGr}(2,4)$ and $\operatorname{LGr}(3,6) \ldots \ldots \ldots$. . . . . . . . . . . . . 29

2. Hypersurfaces in the (real) Lagrangian Grassmannian and second order PDEs. . . . . 30

2.1. Contact manifolds and second order PDEs . . . . . . . . . . . . . . 30

2.2. Nondegenerate second order PDEs and their symbols . . . . . . . . . . . . . . . . . . . 31

$2.3 . \quad$ Symplectic second order PDEs $\ldots \ldots \ldots \ldots$. . . . . . . . . . . . . . . 32

$2.4 . \quad$ The characteristic variety $\ldots \ldots \ldots \ldots \ldots$

2.5. Hyperplane sections and PDEs of Monge-Ampère type. . . . . . . . . . . . . . 34

2.6. Goursat-type Monge-Ampère equations . . . . . . . . . . . . . . . . . . . . . . . . . . . . . 35

2.7. Low-dimensional examples . . . . . . . . . . . . . . . . . . . . . . . 37

2.8. Integrability by the method of hydrodynamic reductions . . . . . . . . . . . 38

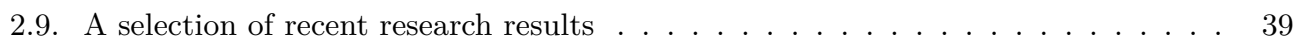

3. Appendix: a guide to reading this volume . . . . . . . . . . . . . . . . . . 40

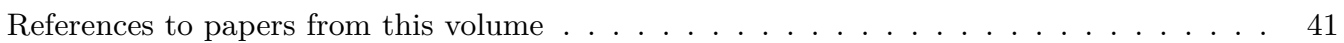

Other references . . . . . . . . . . . . . . . . . . . . . . . . . . . 42

Introduction. One way to see how geometry enters the theory of second order PDEs in one dependent and $n$ independent variables is to regard the Hessian matrix $\left(p_{i j}\right)$ as an $n$-dimensional subspace $\left\langle e_{i}+p_{i j} \epsilon^{j} \mid i=1, \ldots, n\right\rangle$ in the space $\mathbb{R}^{n} \oplus \mathbb{R}^{n *}$. Obviously this correspondence is not accidental: its motivations will be thoroughly surveyed in Section 2 However, a crucial fact can already be noticed: the symmetry of the Hessian matrix corresponds to the subspace $\left\langle e_{i}+p_{i j} \epsilon^{j}\right\rangle$ being isotropic with respect to the canonical symplectic form on $\mathbb{R}^{n} \oplus \mathbb{R}^{n *}$. In other words, the aforementioned subspace is Lagrangian, that is an element of the Lagrangian Grassmannian $\operatorname{LGr}(n, 2 n)$.

The object $\operatorname{LGr}(n, 2 n)$ exists independently of theories of PDEs. It is indeed a very well-known projective variety, displaying a lot of interesting properties, smoothness and homogeneity above all else. As such, it can be studied per se, and this is precisely the purpose of Section 1. Due to the algebro-geometric origin of $\operatorname{LGr}(n, 2 n)$, we shall examine 
the real case (relevant for applications to PDEs) as much as possible in parallel with the complex case.

The entire content of this paper can be found elsewhere scattered throughout the existing literature. Our main goal was to squeeze a plethora of tiny small elements - facts, formulas, lemmas, properties, remarks - into a short self-contained introductory paper. Taken individually they may seem trivial, but their appropriate combination against the motivating background of PDEs form an unexpectedly rich and coherent picture.

The present paper serves yet another purpose. It is included in the Banach Center Publications volume dedicated to the workshop titled Geometry of Lagrangian Grassmannians and Nonlinear PDEs and held in Warsaw in September 2016. The volume is designed in such a way as to provide a source book for a monographic graduate/postgraduate course, as well as a reference for recent research in the discipline (Section 2.9). The present paper may represent a good departing point for the novice. It may also guide the expert finding his/her way in the rest of the volume (see Section 3 .

Background, motivations and acknowledgements. One of the main driving forces behind the present paper, the volume it belongs to and the homonymous workshop has been a conjecture, formulated in 2010 by Ferapontov and his collaborators about the class of second order hydrodynamically integrable PDEs. Essentially, the conjecture states that multidimensional hydrodynamically integrable second order PDEs of Hirota type are of Monge-Ampère type, see [16, Section 1]. Intrigued by Ferapontov's problem, two of us (GM and GM) started a systematic study of the notion of hydrodynamic integrability and soon realised that there was a lot of differential and algebraic geometry at play. More complementary competences were needed. A first informal meeting was held in 2014 in Milan, bringing the problem to the attention of Musso and Russo (both contributors to this volume). Interesting links with the geometry of special projective varieties and homogeneous spaces were highlighted. In 2015 one of us (Moreno) was granted a two-year Maria Skłodowska-Curie Fellowship at IMPAN (Warsaw) for continuing the study of the geometry of hypersurfaces in the Lagrangian Grassmannian and second order PDEs. It was during this period that the authors of the present paper began their cooperation. In 2016 they organised the aforementioned workshop and started editing the present volume.

To date, the conjecture is still open, even though it triggered an enormous amount of side and related works, eventually leading to interesting independent results. The authors wish first of all to thank Professor Ferapontov for his deep and insightful analysis of the phenomenon of hydrodynamic integrability and regret he could not make it to a workshop built, in a sense, around a his idea. Many thanks go also to all the other speakers and contributors to this volume, to Professors: Hwang for his surprise visit, Bryant and Ciliberto for important remarks and valuable advices. The authors thank also Professor Mormul for reviewing the manuscript.

The authors acknowledge the support of the Maria Skłodowska-Curie fellowship SEP210182301 "GEOGRAL", the Institute of Mathematics of the Polish Academy of Sciences, the Banach Centre, the project "FIR (Futuro in Ricerca) 2013-Geometria delle equazioni differenziali", the grant 346300 for IMPAN from the Simons Foundation and the matching 2015-2019 Polish MNiSW fund. Giovanni Moreno has been also partially founded by the 
Polish National Science Centre grant under the contract number 2016/22/M/ST1/00542. Gianni Manno was partially supported by a "Starting Grant per Giovani Ricercatori" 53_RSG16MANGIO of the Polytechnic of Turin. Gianni Manno and Giovanni Moreno are members of G.N.S.A.G.A of I.N.d.A.M.

\section{Geometry of the (real and complex) Lagrangian Grassmannian}

1.1. Preliminaries. One of the harshest lessons from earlier studies in Mathematics is the impossibility to identify a vector space with its dual in a canonical way. This is mirrored in Physics by the profound difference between vectors and covectors. The former correspond geometrically to those fancy arrows emanating from 0 , whereas the latter are hyperplanes passing through 0 .

However, if a "balanced mixture" of vectors and covectors is given, such as in the space $V \oplus V^{*}$, then there is an obvious way to identify the space with its dual. Just perform a "counterclockwise rotation by $\frac{\pi}{2}$ ", having identified the horizontal axis with $V$ and the vertical axis with $V^{*}$. The evident analogy with the multiplication by $i$ in the complex plane led to the coinage of the term symplectic by Hermann Weyl in 1939 [47, page 165]. Indeed the preposition "sym" is the Greek analog of the Latin preposition "cum", see, e.g., [10, pp. xiii-xiv] and [39].

From now on, $V$ is a linear vector space of dimension $n$, and $\omega$ is the 2 -form on $V \oplus V^{*}$ corresponding to the canonical identification $\left(V \oplus V^{*}\right)^{*} \equiv V \oplus V^{*}$. The pair $\left(V \oplus V^{*}, \omega\right)$ is, up to equivalences, the unique linear symplectic space of dimension $2 n$. When coordinates are required, we fix a basis $\left\{e_{i}\right\}_{i=1, \ldots, n}$ in $V$ and we consider its dual $\left\{\epsilon^{i}\right\}_{i=1, \ldots, n}$ in $V^{*}$. If the results do not depend on the ground field, we leave it unspecified - that is, it may be either $\mathbb{R}$ or $\mathbb{C}$.

In the above coordinates, the matrix of $\omega$ is

$$
I:=\left(\begin{array}{cc}
0 & -\mathrm{Id} \\
\mathrm{Id} & 0
\end{array}\right),
$$

and it is known as the standard symplectic matrix. Indeed,

$$
\begin{gathered}
V \oplus V^{*} \stackrel{\omega}{\longrightarrow} V^{*} \oplus V=\left(V \oplus V^{*}\right)^{*}, \\
v+\alpha \longmapsto-\alpha+v,
\end{gathered}
$$

with respect to the bases $\left(e_{1}, \ldots, e_{n}, \epsilon^{1}, \ldots, \epsilon^{n}\right)$ and $\left(\epsilon^{1}, \ldots, \epsilon^{n}, e_{1}, \ldots, e_{n}\right)$ of $V \oplus V^{*}$ and $V^{*} \oplus V$, respectively.

We stress that (1) is not a rotation matrix - it is the matrix corresponding to the two-form

$$
\omega=\epsilon^{i} \wedge e_{i} .
$$

Observe that in (2), as well as in the rest of the paper, we use the Einstein convention for repeated indexes, unless otherwise specified.

1.2. Definition of the Lagrangian Grassmannian. It is well-known that the set

$$
\operatorname{Gr}\left(n, V \oplus V^{*}\right):=\left\{L \subset V \oplus V^{*} \mid L \text { linear subspace, } \operatorname{dim} L=n\right\}
$$

possesses the structure of an $n^{2}$-dimensional smooth manifold, known as the Grassmannian (manifold/variety) (see, e.g., [23, Lecture 6] for an algebro-geometric proof or 
[33. Lemma 5.1] for a differential-geometric proof). The key is the injective map 1

$$
\begin{gathered}
V^{*} \otimes V^{*}=\operatorname{Hom}\left(V, V^{*}\right) \longrightarrow \operatorname{Gr}\left(n, V \oplus V^{*}\right), \\
h \longmapsto \arctan h:=\langle v+h(v) \mid v \in V\rangle,
\end{gathered}
$$

which allows one to define an $n^{2}$-dimensional chart in $\operatorname{Gr}\left(n, V \oplus V^{*}\right)$. This chart is also dense - whence the name big cell which we shall use from now on.

If $h=h_{i j} \epsilon^{i} \otimes \epsilon^{j}$, then

$$
\arctan h:=\left\langle e_{i}+h_{i j} \epsilon^{j} \mid i=1, \ldots, n\right\rangle .
$$

Let us impose that $\arctan h$ be isotropic with respect to the two-form $\omega$, that is

$$
\left.\omega\right|_{\arctan h} \equiv 0 \text {. }
$$

In view of (5), condition (6) reads

$$
\omega\left(e_{i_{1}}+h_{i_{1} j_{1}} \epsilon^{j_{1}}, e_{i_{2}}+h_{i_{2} j_{2}} \epsilon^{j_{2}}\right)=0, \quad \forall i_{1}, i_{2}=1, \ldots, n .
$$

Since $\omega\left(e_{i_{1}}+h_{i_{1} j_{1}} \epsilon^{j_{1}}, e_{i_{2}}+h_{i_{2} j_{2}} \epsilon^{j_{2}}\right)=h_{i_{1} i_{2}}-h_{i_{2} i_{1}}$, it is obvious that 6 is fulfilled if and only if the matrix $h_{i j}$ is symmetric, that is $h \in S^{2} V^{*}$.

The Lagrangian Grassmannian (manifold/variety) $\operatorname{LGr}\left(n, V \oplus V^{*}\right)$ can be defined as the closure of the subset $S^{2} V^{*}$ of the big cell $V^{*} \otimes V^{*}$. From this point of view, $\operatorname{LGr}\left(n, V \oplus V^{*}\right)$ is a compactification of the space of symmetric forms on $V$. In the geometric theory of PDEs based on jet spaces [28], the additional "points at infinity" correspond to the so-called singularities of solutions [46, Section 2.2], see Fig. 1 .

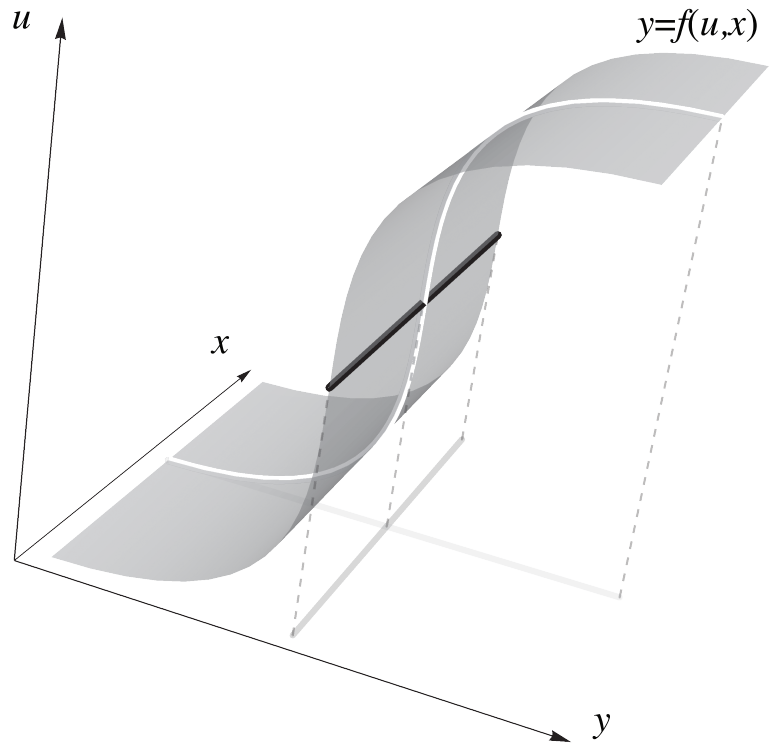

Fig. 1. In the framework of contact geometry, the distinction between dependent $(u)$ and independent $(x, y)$ variables simply disappears. The smooth surface depicted here- the graph of $y=f(u, x)$, with $f$ smooth - cannot be interpreted as a "regular" solution in the sense of a function $u=u(x, y)$. There is a locus, highlighted as a thick black line, where the tangent planes to

\footnotetext{
${ }^{1}$ Observe that $\arctan h$ is nothing but the graph of $h$. The symbol "arctan" has been chosen in order to be consistent with Smith's contribution to this very volume, see [T3, Section 2].
} 
the surface project degenerately to the $(x, y)$-plane (e.g., the projection of the thick white curve crosses the projection of the thick black line at zero speed, regardlessly of its parametrisation). Such a locus is called a singularity of the solution. We warn the reader that the terminology is misleading, since the surface is perfectly smooth.

From now on, the open subset

$$
S^{2} V^{*} \subset \operatorname{LGr}\left(n, V \oplus V^{*}\right)
$$

will be referred to as the big cell of the Lagrangian $\operatorname{Grassmannian} \operatorname{LGr}\left(n, V \oplus V^{*}\right)$.

1.3. Coordinate-free definition of the Lagrangian Grassmannian. On a deeper conceptual level, the symplectic form $\omega$ can be found by decomposing the space of twoforms on $V \oplus V^{*}$ into $\mathrm{GL}(V)$-irreducible representations:

$$
\wedge^{2}\left(V \oplus V^{*}\right)=\wedge^{2} V \oplus\left(V \otimes V^{*}\right) \oplus \wedge^{2} V^{*} .
$$

Then $\omega$ is precisely the element of the left-hand side corresponding to the element $0+\mathrm{id}+0$ of the right-hand side. Then one can set

$$
\operatorname{LGr}\left(V \oplus V^{*}\right):=\left\{L \subset V \oplus V^{*} \mid L \text { linear subspace, } \operatorname{dim} L=n,\left.\omega\right|_{L} \equiv 0\right\} .
$$

1.4. The Plücker embedding. While $S^{2} V^{*}$ provides a convenient local description of $\operatorname{LGr}\left(V \oplus V^{*}\right)$, the rich global geometry of $\operatorname{LGr}\left(V \oplus V^{*}\right)$ is invisible from the point of view of the big cell. Global features become evident when the object is embedded into a "flat" environment. In the present case the role of such an environment is played by an appropriate projective subspace of $\mathbb{P}\left(\wedge^{n}\left(V \oplus V^{*}\right)\right)$.

The trick to obtain the desired embedding consists in regarding an $n$-dimensional subspace $L \in \operatorname{LGr}\left(V \oplus V^{*}\right)$ as a line in $\wedge^{n}\left(V \oplus V^{*}\right)$. Indeed, a basis $\left\{l_{1}, \ldots, l_{n}\right\}$ of $L$ defines, up to a projective factor, a unique (nonzero) $n$-vector $l_{1} \wedge \ldots \wedge l_{n}$. The projective class of the latter is then unambiguously associated with $L$, and we will call it the volume of $L$ and denote it by $\operatorname{vol}(L)$.

The map

$$
\begin{aligned}
\operatorname{LGr}\left(V \oplus V^{*}\right) & \longrightarrow \mathbb{P}\left(\wedge^{n}\left(V \oplus V^{*}\right)\right), \\
L & \longmapsto \operatorname{vol}(L),
\end{aligned}
$$

is called the Plücker embedding. The basic properties of (11) are easily checked.

First, the element $\operatorname{vol}(L)$ is represented by a decomposable $n$-vector, that is an $n$-vector $\xi$ satisfying the equation $\xi \wedge \xi=0$. The latter is a quadratic condition, symmetric for $n$ even and skew-symmetric for $n$ odd.

Second, the representative $\xi$ is transversal to $\omega$, in the sense that

$$
\left.\iota_{\omega}(\xi):=\omega\right\lrcorner \xi=0 .
$$

This means that, in fact $\xi$ belongs to the linear subspace

$$
\operatorname{ker} \iota_{\omega}=\operatorname{ker}\left(\wedge^{n}\left(V \oplus V^{*}\right) \stackrel{\iota_{\omega}}{\longrightarrow} \wedge^{n-2}\left(V \oplus V^{*}\right)\right)
$$

of $\wedge^{n}\left(V \oplus V^{*}\right)$.

Third, 11 is injective. 
1.5. The Plücker embedding space. The fact that ker $\iota_{\omega}$ is not the smallest linear subspace of $\wedge^{n}\left(V \oplus V^{*}\right)$ whose projectivization contains the image of (11) is less evident and requires more care.

To this end, observe that

$$
\wedge^{n}\left(V \oplus V^{*}\right)=\bigoplus_{i=0}^{n} \wedge^{i}\left(V^{*}\right) \otimes \wedge^{n-i}(V) \simeq \bigoplus_{i=0}^{n} \wedge^{i}\left(V^{*}\right) \otimes \wedge^{i}\left(V^{*}\right),
$$

in view of the Poincaré duality $\wedge^{n-i}(V)=\operatorname{Hom}\left(\wedge^{i}(V), \wedge^{n}(V)\right)$. It is then easy to realise that the representative $\xi$ of $\operatorname{vol}(L)$ belongs to

$$
\bigoplus_{i=0}^{n} S^{2}\left(\wedge^{i}\left(V^{*}\right)\right)
$$

via the map (11). Puzzlingly enough, (15) is not yet the minimal subspace we were looking for. Though it is so for $n=2,3$. Let $n=2$ and let $L$ be the Lagrangian 2-plane (5) corresponding to the symmetric $2 \times 2$ matrix $\left(h_{i j}\right)$. Then $\operatorname{vol}(L)=[\xi]$, with

$$
\xi=e_{1} \wedge e_{2}+h_{11} e_{1} \wedge \epsilon^{1}+h_{12} e_{1} \wedge \epsilon^{2}+h_{21} e_{2} \wedge \epsilon^{1}+h_{22} e_{2} \wedge \epsilon^{2}+\operatorname{det}\left(h_{i j}\right) \epsilon^{1} \wedge \epsilon^{2}
$$

as an element of $\wedge^{2}\left(V \oplus V^{*}\right)$, and

$$
\xi=1+h_{i j} \epsilon^{i} \odot \epsilon^{j}+\operatorname{det}\left(h_{i j}\right) \epsilon^{1} \wedge \epsilon^{2}
$$

as an element of 15 . That is,

$$
\left(1, h_{11}, h_{12}, h_{22}, h_{11} h_{22}-h_{12}^{2}\right)
$$

are the coordinates of $\xi$ in the standard basis of $(15)$. Observe that in $(18)$ there appear all the minors of the matrix $\left(h_{i j}\right)$, namely: minors of order 0 (the constant 1 ), minors of order 1 (the very entries of the matrix) and minors of order 2 (the determinant).

Similarly, for $n=3$, one finds

$$
\xi=1+h_{i j} \epsilon^{i} \odot \epsilon^{j}+h_{i j}^{\#}\left(\epsilon^{1} \wedge \widehat{\epsilon^{i}} \wedge \epsilon^{3}\right) \odot\left(\epsilon^{1} \wedge \widehat{\epsilon^{j}} \wedge \epsilon^{3}\right)+\operatorname{det}\left(h_{i j}\right) \epsilon^{1} \wedge \epsilon^{2} \wedge \epsilon^{3},
$$

where $\left(h_{i j}^{\#}\right)$ denotes the cofactor matrix of $\left(h_{i j}\right)$ and the hat indicates a removed element. One gets then the 14 coordinates

$$
\left(1, h_{11}, h_{12}, h_{13}, h_{22}, h_{23}, h_{33}, h_{11}^{\#}, h_{12}^{\#}, h_{13}^{\#}, h_{22}^{\#}, h_{23}^{\#}, h_{33}^{\#}, \operatorname{det}\left(h_{i j}\right)\right)
$$

corresponding to the point $\xi$.

It is then easy to realise that, in the case $n=4$, one has

$$
\left(1, \ldots, h_{i j}, \ldots, 2 \times 2 \text { minors }, \ldots, h_{i j}^{\#}, \ldots, \operatorname{det}\left(p_{i j}\right)\right) .
$$

A $4 \times 4$ symmetric matrix contains exactly: 1 minor of order 0 and of order $4, \frac{4 \cdot 5}{2}=10$ minors of order 1 and $3, \frac{6 \cdot 7}{2}=21$ minors of order 2 , where $6=\left(\begin{array}{l}4 \\ 2\end{array}\right)$ is the number of choices of 2 rows (columns). Therefore, (21) consists exactly of $1+10+21+10+1=43$ entries. The subtle point here is that, as opposed to the cases $n=2$ and $n=3$, not all the minors are linearly independent. More precisely there is exactly one linear combination of $2 \times 2$ minors, namely

$$
-\left(h_{13} h_{24}-h_{14} h_{23}\right)+\left(h_{12} h_{34}-h_{14} h_{23}\right)-\left(h_{12} h_{34}-h_{13} h_{24}\right),
$$


which vanishes [35. Therefore, there is a (proper, for $n \geq 4$ ) linear subspace of (15), henceforth denoted by

$$
\wedge_{0}^{n}\left(V \oplus V^{*}\right):=\bigoplus_{i=0}^{n} S_{0}^{2}\left(\wedge^{i}\left(V^{*}\right)\right),
$$

which contains all the $\xi$ 's and it is minimal with respect to this property. Summing up,

$$
\begin{aligned}
n=2 \Rightarrow & \wedge_{0}^{2}\left(V \oplus V^{*}\right)=\wedge^{0}\left(V^{*}\right) \oplus S^{2}\left(V^{*}\right) \oplus \wedge^{2}\left(V^{*}\right) \text { has dimension 5, } \\
n=3 \Rightarrow & \wedge_{0}^{3}\left(V \oplus V^{*}\right)=\wedge^{0}\left(V^{*}\right) \oplus S^{2}\left(V^{*}\right) \oplus S^{2}\left(\wedge^{2}\left(V^{*}\right)\right) \oplus \wedge^{3}\left(V^{*}\right) \text { has dimension 14, } \\
n=4 \Rightarrow & \wedge_{0}^{4}\left(V \oplus V^{*}\right)=\wedge^{0}\left(V^{*}\right) \oplus S^{2}\left(V^{*}\right) \oplus S_{0}^{2}\left(\wedge^{2}\left(V^{*}\right)\right) \oplus S^{2}\left(\wedge^{3}\left(V^{*}\right)\right) \oplus \wedge^{4}\left(V^{*}\right) \\
& \text { has dimension } 42 .
\end{aligned}
$$

Therefore, the minimal projective embedding of the 3-(resp., 6- and 10-)dimensional Lagrangian Grassmannian $\operatorname{LGr}(2,4)$ (resp., $\operatorname{LGr}(3,6)$ and $\operatorname{LGr}(4,8)$ ) is $\mathbb{P}^{4}$ (resp., $\mathbb{P}^{13}$ and $\left.\mathbb{P}^{41}\right)$.

In general, to find the Plücker embedding space of the $\frac{n(n+1)}{2}$-dimensional Lagrangian Grassmannian $\operatorname{LGr}(n, 2 n)$, one has to count how many minors a symmetric $n \times n$ matrix possesses, minors of order 0 and $n$ included. This, in principle, is an easy task. The problem is to look for dependencies of the form (22) among minors. The number of minors needs to be diminished by the number of these relations. The result, further decreased by one, represents the (projective) dimension of the sought-for space. In Section 1.12 below we explain how the very same space can be obtained by exploiting the theory of representations.

1.6. The Plücker relations. Expressions (18), 20 and (21) represent the parametric description of $\operatorname{LGr}(2,4), \operatorname{LGr}(3,6)$ and $\operatorname{LGr}(4,8)$ in $\mathbb{P}^{4}, \mathbb{P}^{13}$ and $\mathbb{P}^{41}$, respectively. Let us denote by

$$
\left[z_{0}: z_{1}: \ldots: z_{N}\right]
$$

the standard projective coordinates on $\mathbb{P}^{N}$. Then it is easy to realise that points of $\operatorname{LGr}(2,4)$ satisfy the quadratic relation

$$
z_{1} z_{3}-z_{2}^{2}-z_{0} z_{4}=0
$$

capturing the fact that, on $\operatorname{LGr}(2,4)$, the fourth coordinate is the determinant of the symmetric matrix whose entries are $z_{1}, z_{2}$ and $z_{3}$. Observe that $z_{0}$, which is 1 on the big cell of $\operatorname{LGr}(2,4)$, serves the sole purpose of homogenising the relation

$$
z_{4}=\operatorname{det}\left(\begin{array}{cc}
z_{1} & z_{2} \\
z_{2} & z_{3}
\end{array}\right) .
$$

Indeed, 25 is the equation cutting out $\operatorname{LGr}(2,4)$ in $\mathbb{P}^{4}$. Therefore, $\operatorname{LGr}(2,4)$ is a quadric.

The codimension of $\operatorname{LGr}(3,6)$ in $\mathbb{P}^{13}$, on the contrary, is quite high: 7 . The corresponding equations are essentially the Laplace rule for the determinant of a symmetric $3 \times 3$ matrix. More precisely, if

$$
Z:=\left(\begin{array}{ccc}
z_{1} & z_{2} & z_{3} \\
z_{2} & z_{4} & z_{5} \\
z_{3} & z_{5} & z_{6}
\end{array}\right)
$$


then the seven quadratic equations

$$
\begin{aligned}
z_{0} z_{7} & =Z_{11}^{\#} \\
z_{0} z_{8} & =Z_{12}^{\#} \\
z_{0} z_{9} & =Z_{13}^{\#} \\
z_{0} z_{10} & =Z_{22}^{\#} \\
z_{0} z_{11} & =Z_{23}^{\#} \\
z_{0} z_{12} & =Z_{33}^{\#} \\
z_{0} z_{13} & =\operatorname{tr}\left(Z \cdot Z^{\#}\right)
\end{aligned}
$$

cut out $\operatorname{LGr}(3,6)$ in $\mathbb{P}^{13}$. Similarly, it can be proved that $\operatorname{LGr}(n, 2 n)$ is cut out by quadratic relations in its own Plücker embedding space $\mathbb{P}\left(\wedge_{0}^{n}\left(V \oplus V^{*}\right)\right)$, similar to 25 and the seven equations above (see, e.g., [32, Theorem 14.6]). These relations are usually referred to as the Plücker relations, whereas the expressions (18), 20, 21, as well as the analogous ones for higher values of $n$, are called the Plücker coordinates of the point $L \in \operatorname{LGr}(n, 2 n)$.

1.7. The dual variety. The case of $\operatorname{LGr}(2,4)$ is somewhat special in that the dimension of the Plücker embedding space $\mathbb{P}^{4}$ exceeds only by one the dimension of $\operatorname{LGr}(2,4)$. Then the tangent spaces to $\operatorname{LGr}(2,4)$ are projective hyperplanes in $\mathbb{P}^{4}$. The latter form a set, usually denoted by $\mathbb{P}^{4 *}$ and called the dual of $\mathbb{P}^{4}$, which is (non-canonically) identified with $\mathbb{P}^{4}$ itself. Thus, the set of tangent hyperplanes to $\operatorname{LGr}(2,4)$ constitutes a subset

$$
\operatorname{LGr}(2,4)^{*} \subseteq \mathbb{P}^{4 *}
$$

of the set of all hyperplanes, accordingly called the dual variety of $\operatorname{LGr}(2,4)$.

We warn the reader that an element $\pi \in \operatorname{LGr}(2,4)^{*}$ is a linearly embedded $\mathbb{P}^{3}$, which has contact of order one in some point with $\operatorname{LGr}(2,4)$. In the language of jets,

$$
\operatorname{LGr}(2,4)^{*}=\left\{\pi \in \mathbb{P}^{4 *} \mid j_{x}^{1}(\pi)=j_{x}^{1}(\operatorname{LGr}(2,4)) \text { in some point } x \in \operatorname{LGr}(2,4)\right\} .
$$

Therefore, the same $\pi$, which is tangent at $x \in \operatorname{LGr}(2,4)$, may intersect unpredictably $\operatorname{LGr}(2,4)$ someplace else. In fact, as we shall see later on, elements of $\operatorname{LGr}(2,4)^{*}$ allow constructing special hypersurfaces in $\operatorname{LGr}(2,4)$ called hyperplane sections. Hence, the notion of an element of the dual variety is different from (though related to) the notion of a tangent space to $\operatorname{LGr}(2,4)$, that is a fibre of an abstract linear bundle of rank 3 .

Another peculiarity of $\operatorname{LGr}(2,4)$ is that - exceptionally among all Lagrangian Grassmannians - its dual variety is smooth and canonically isomorphic to $\operatorname{LGr}(2,4)$ itself. That is, it is cut by the very same equation (25), appropriately interpreted as an equation in $\mathbb{P}^{4 *}$. This also follows from the fact that $\operatorname{LGr}(2,4)$ has codimension one in $\mathbb{P}^{4}$, and that $\operatorname{LGr}(2,4)$ is smooth. Indeed, to any point $x \in \operatorname{LGr}(2,4)$ one associates the unique element $\pi_{x} \in \mathbb{P}^{4 *}$ which is tangent to $\operatorname{LGr}(2,4)$ at $x$. This realises the desired one-to-one correspondence.

The case of $\operatorname{LGr}(3,6)$ is already much more involved. Indeed, at any $x \in \operatorname{LGr}(3,6)$ there is certainly a unique tangent 6 -dimensional subspace but there does not need to be a unique tangent hyperplane (i.e., a 12-dimensional subspace). Actually, there is a 6-dimensional family of them, making the dual variety $\operatorname{LGr}(3,6)^{*} 12$-dimensional. It can be proved that it is cut out by a single quartic relation in $\mathbb{P}^{13 *}[\mathrm{T4}$, Section 5]. 
There is still a certain correspondence between $\operatorname{LGr}(3,6)$ and its dual $\operatorname{LGr}(3,6)^{*}$. The former is isomorphic to the singular locus ${ }^{2}$ of the latter, viz.

$$
\operatorname{LGr}(3,6) \equiv \operatorname{Sing}\left(\operatorname{LGr}(3,6)^{*}\right) .
$$

This should help to convince oneself of the validity of 30 . Let us describe an element $\pi \in \mathbb{P}^{14 *}$ by projective coordinates

$$
\pi \equiv\left[A: \ldots: B^{i j}: \ldots: \ldots: C^{i j}: \ldots: D\right] .
$$

Then, the intersection $\pi \cap \operatorname{LGr}(3,6)$, in the Plücker coordinates $(20)$, is given by

$$
A+B^{i j} h_{i j}+C^{i j} h_{i j}^{\#}+D \operatorname{det}\left(h_{i j}\right)=0 .
$$

The key remark is that a particular case of an expression of the form 32 can be obtained by means of another symmetric $3 \times 3$ matrix, say $H=\left(H_{i j}\right)$. More precisely,

$$
\operatorname{det}(h-H)=0
$$

is a particular form of equation (32) above, where the fourteen coefficients $A, B^{i j}, C^{i j}, D$ depend on the six coefficients $H_{i j}$. It is not hard to realise that, after the substitutions

$$
A:=\operatorname{det} H, \quad B:=H^{\#}, \quad C:=H, \quad D:=1,
$$

the equation $(32)$ becomes $(33)$. On the top of that, the hyperplane $\pi_{H}$, with coefficients given by (34) is tangent to $\operatorname{LGr}(3,6)$. This is not hard to see: the left-hand side of (33), regarded as a function of $h$, vanishes at $h=H$, together with its first derivatives.

In other words $\pi \in \operatorname{LGr}(3,6)^{*}$ because $\pi_{H}$ is tangent to $\operatorname{LGr}(3,6)$ at the point $x_{H}$ given, in the coordinates (20), by $H$ itself. The correspondence

$$
\pi_{H} \longmapsto x_{H}
$$

basically allows us to regard the same matrix $H$ as a special element $\pi_{H}$ of $\operatorname{LGr}(3,6)^{*}$ as well as an element $x_{H}$ of $\operatorname{LGr}(3,6)$ itself, thus realising the desired isomorphism 30 .

The duality (35) manifests itself for any $\operatorname{LGr}(n, 2 n)$, though the isomorphism 30 now must be recast as

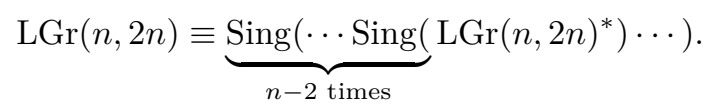

The underlying structure responsible of this duality is the natural bilinear form

$$
\begin{gathered}
\wedge_{0}^{n}\left(V \oplus V^{*}\right) \times \wedge_{0}^{n}\left(V \oplus V^{*}\right) \longrightarrow \wedge^{2 n}\left(V \oplus V^{*}\right), \\
(\alpha, \beta) \longmapsto \alpha \wedge \beta,
\end{gathered}
$$

which is scalar-valued and is symmetric (resp., skew-symmetric) for $n$ even (resp., odd). Indeed, the above-defined bilinear form is non-degenerate, thus allowing a point-hyperplane correspondence in the (de-projectivised) Plücker embedding space $\wedge_{0}^{n}\left(V \oplus V^{*}\right)$. After projectivisation, this correspondence coincides precisely with (35). It is interesting to notice that such a correspondence is equivalent to the fact that the cone over $\operatorname{LGr}(n, 2 n)$ be isotropic with respect to (37).

\footnotetext{
${ }^{2}$ By singular locus of an algebraic variety $X=\left\{f_{1}=0, \ldots, f_{m}=0\right\}$ of codimension $m$ we mean the subset of $X$ where the differentials $d f_{1}, \ldots, d f_{m}$ are not linearly independent.
} 
The dual $\mathbb{P}\left(\wedge_{0}^{n}\left(V \oplus V^{*}\right)\right)^{*}$ of the Plücker embedding space parametrises the hyperplane sections of the Lagrangian Grassmannian, which correspond to the so-called MongeAmpère equations, see Section 2.5 below. The stratification of $\mathbb{P}\left(\wedge_{0}^{n}\left(V \oplus V^{*}\right)\right)^{*}$ by the dual variety $\operatorname{LGr}(n, 2 n)^{*}$ and its singular loci will correspond to special $\left(\mathrm{Sp}_{2 n}\right.$-invariant, see next Section 1.8) classes of such PDEs (see Section 2.7).

The study of these special classes of PDEs corresponds precisely to the study of the orbits in $\mathbb{P}\left(\wedge_{0}^{n}\left(V \oplus V^{*}\right)\right)^{*}$ of the natural groups acting on $\wedge_{0}^{n}\left(V \oplus V^{*}\right)$ (see Section 1.8 below). In particular, there is a unique close orbit with respect to the symplectic group, and this is precisely the "very singular" locus $\operatorname{LGr}(n, 2 n)$ (see Section 1.9).

1.8. Natural group actions on $\operatorname{LGr}\left(V \oplus V^{*}\right)$. Recall that the "arctangent map" (4) allowed us to define a canonical embedding of $S^{2} V^{*}$ into the Lagrangian Grassmannian $\operatorname{LGr}\left(V \oplus V^{*}\right)$, whose image corresponds to the big cell (8). Let us further restrict our scope by considering only the non-degenerate elements of the big cell. That is, the open subset

$$
\mathcal{U}:=\left\{h \in S^{2} V^{*} \mid \operatorname{det}(h) \neq 0\right\} \subset \operatorname{LGr}\left(V \oplus V^{*}\right) .
$$

One obvious group action on $\mathcal{U}$ is easily found. Indeed, any element $D \in \mathrm{GL}(V)$ acts naturally on symmetric forms,

$$
h \longmapsto D^{t} \cdot h \cdot D,
$$

where the same symbol $h$ denotes both the matrix and the form itself. As a matter of fact, 38 acts on the whole big cell, preserving the subset $\mathcal{U}$.

Another group action on $\mathcal{U}$ is due to the linear structure of the big cell. Indeed, an element $C$ of the Abelian group $S^{2} V^{*}$ can act on $S^{2} V^{*}$ itself as a translation:

$$
h \longmapsto h+C \text {. }
$$

Observe that, unlike (38), the action 39 does not preserves $\mathcal{U}$.

One last, somewhat less evident, group action on $\mathcal{U}$ is given by

$$
h \longmapsto h \cdot(\operatorname{Id}+B \cdot h)^{-1},
$$

where now $B \in S^{2} V$. Above correspondence (40) can be explained as follows. There is an analogue of the arctangent map (4), defined on $S^{2} V$, instead of $S^{2} V^{*}$, that is

$$
S^{2} V \ni k \longmapsto \underline{\arctan }(k):=\left\langle\alpha+k(\alpha) \mid \alpha \in V^{*}\right\rangle .
$$

Observe that the common image of arctan and $\underline{\arctan }$ is precisely $\mathcal{U}$. Therefore, 400 is nothing but the translation

$$
h^{-1} \longmapsto h^{-1}+B
$$

by $B$ of $h^{-1}$, understood as an element of $S^{2} V$ via arctan. Indeed,

$$
\begin{aligned}
\underline{\arctan } & \left(h^{-1}+B\right)=\left\langle\alpha+\left(h^{-1}+B\right)(\alpha) \mid \alpha \in V^{*}\right\rangle \\
& =\left\langle h(v)+\left(h^{-1}+B\right)(h(v)) \mid v \in V\right\rangle=\langle h(v)+(\operatorname{Id}+B \cdot h)(v) \mid v \in V\rangle \\
& =\left\langle h\left((\operatorname{Id}+B \cdot h)^{-1}(v)\right)+(\operatorname{Id}+B \cdot h)\left((\operatorname{Id}+B \cdot h)^{-1}(v)\right) \mid v \in V\right\rangle \\
& =\left\langle v+\left(h(\operatorname{Id}+B \cdot h)^{-1}\right)(v) \mid v \in V\right\rangle=\arctan \left(h \cdot(\operatorname{Id}+B \cdot h)^{-1}\right),
\end{aligned}
$$

where we used the facts that $h$ is invertible and that, at least locally around $0, \operatorname{Id}+B \cdot h$ is invertible as well. 
The three actions (38), (39) and (40) above may seem accidental and unrelated. On the contrary, they share a common background. Consider a linear transformation of $V \oplus V^{*}$, represented, in the aforementioned basis, by the $(2 n) \times(2 n)$ matrix

$$
M:=\left(\begin{array}{cc}
A & B \\
C & D
\end{array}\right) .
$$

In the same basis, the $(2 n) \times n$ matrix

$$
\left(\begin{array}{c}
\operatorname{Id}_{n} \\
h
\end{array}\right) \equiv \arctan (h)
$$

represents the $n$-dimensional linear subspace $\arctan (h)$. Indeed, the $n$ columns of the matrix (44) corresponds to the $n$ generators appearing in the definition (5) of $\arctan (h)$. Observe that

$$
\left(\begin{array}{c}
\operatorname{Id}_{n} \\
h
\end{array}\right), \quad\left(\begin{array}{c}
A \\
h \cdot A
\end{array}\right)
$$

represent the same subspace, for any $A \in \mathrm{GL}(V)$.

We need now to make the crucial assumption that $M$ belongs to a small neighbourhood of the identity. This allows us to act by $M$ on $\arctan (h)$ as follows:

$$
\begin{aligned}
M \cdot \arctan (h) & =\left(\begin{array}{ll}
A & B \\
C & D
\end{array}\right) \cdot\left(\begin{array}{c}
\operatorname{Id}_{n} \\
h
\end{array}\right) \\
& =\left(\begin{array}{c}
A+B \cdot h \\
C+D \cdot h
\end{array}\right)=\left(\begin{array}{c}
\operatorname{Id}_{n} \\
(C+D \cdot h)(A+B \cdot h)^{-1}
\end{array}\right) \\
& =\arctan \left((C+D \cdot h)(A+B \cdot h)^{-1}\right) .
\end{aligned}
$$

Directly from the definition of a Lagrangian subspace of $V \oplus V^{*}$ it follows that $(C+D \cdot h)(A+B \cdot h)^{-1}$ is again a symmetric form if and only if the transformation $M$ preserves the symplectic form $\omega$, that is,

$$
M^{t} \cdot I \cdot M=I,
$$

where $I$ is the symplectic matrix (1). A matrix $M$ fulfilling (47) is called symplectic transformation. The three matrices

$$
\left(\begin{array}{cc}
\left(D^{t}\right)^{-1} & 0 \\
0 & D
\end{array}\right), \quad\left(\begin{array}{cc}
1 & 0 \\
C & 1
\end{array}\right), \quad\left(\begin{array}{ll}
1 & B \\
0 & 1
\end{array}\right),
$$

with $D \in \mathrm{GL}(V), C \in S^{2} V^{*}$ and $B \in S^{2} V$ are easily checked to satisfy (47) and they correspond to the actions 38, , 39) and (40), respectively.

Actually, the three matrices 48 generate the entire subgroup

$$
\mathrm{Sp}_{2 n} \equiv \mathrm{Sp}\left(V \oplus V^{*}\right) \subset \mathrm{GL}\left(V \oplus V^{*}\right)
$$

of symplectic transformations, that is what is usually called the symplectic group. Such an "inner structure" of the symplectic group becomes even more evident on the level of the corresponding Lie algebras, viz.

$$
\mathfrak{s p}_{2 n} \equiv \mathfrak{s p}\left(V \oplus V^{*}\right)=S^{2} V^{*} \oplus \mathfrak{g l}(V) \oplus S^{2} V .
$$

This structure is the source of all the structures on $\operatorname{LGr}\left(V \oplus V^{*}\right)$ we shall find later on. The homogeneous one, to begin with. 
1.9. The homogeneous structure of $\operatorname{LGr}\left(V \oplus V^{*}\right)$. Formula 46 immediately shows that

$$
M \cdot \arctan (0)=\arctan (0) \Leftrightarrow A=\operatorname{Id}_{n}, C=0 .
$$

In other words, the stabiliser subgroup

$$
P:=\operatorname{Stabsp}_{2 n}(\arctan (0))=\left\{\left(\begin{array}{cc}
\operatorname{Id}_{n} & B \\
0 & D
\end{array}\right) \mid B \in S^{2} V, D \in \mathrm{GL}(V)\right\},
$$

which coincides with the semidirect product

$$
P=\mathrm{GL}(V) \rtimes S^{2} V,
$$

encompasses the transformations of the form (38) and 40). Those of the form (39), that is $S^{2} V^{*}$ acting by translations on the big cell $S^{2} V^{*}$, clearly allow us to move the origin $\arctan (0)$ into any other point $\arctan (h)$ of the big cell. So, the orbit $\mathrm{Sp}_{2 n} / P$ contains the big cell $S^{2} V^{*}$. However, since $\mathrm{Sp}_{2 n}$ is compact and $P$ is closed, it must be

$$
\mathrm{Sp}_{2 n} / P=\overline{S^{2} V^{*}}=\operatorname{LGr}(n, 2 n) .
$$

That is, the $\mathrm{Sp}_{2 n}$-action is transitive and $\operatorname{LGr}(n, 2 n)$ is a homogeneous space of the Lie group $\mathrm{Sp}_{2 n}$.

Having ascertained the transitivity of the $\mathrm{Sp}_{2 n}$-action, we can switch to the local point of view and analyse the infinitesimal action of $\mathfrak{s p}_{2 n}$. Assume that

$$
M_{\epsilon}=\left(\begin{array}{cc}
A_{\epsilon} & B_{\epsilon} \\
C_{\epsilon} & D_{\epsilon}
\end{array}\right)
$$

passes through the identity at $\epsilon=0$, and differentiate formula 46 :

$$
\begin{aligned}
& \left.\frac{\mathrm{d}}{\mathrm{d} \epsilon}\right|_{\epsilon=0} M_{\epsilon} \cdot h=\left.\frac{\mathrm{d}}{\mathrm{d} \epsilon}\right|_{\epsilon=0}\left(C_{\epsilon}+D_{\epsilon} \cdot h\right)\left(A_{\epsilon}+B_{\epsilon} \cdot h\right)^{-1} \\
& =\left(\dot{C}_{0}+\dot{D}_{0} \cdot h\right)\left(A_{0}+B_{0} \cdot h\right)^{-1}-\left(C_{0}+D_{0} \cdot h\right)\left(\dot{A}_{0}+\dot{B}_{0} \cdot h\right)\left(A_{0}+B_{0} \cdot h\right)^{-2} \\
& =\dot{C}_{0}+\dot{D}_{0} \cdot h-h \cdot\left(\dot{A}_{0}+\dot{B}_{0} \cdot h\right),
\end{aligned}
$$

as well as 47):

$$
\begin{aligned}
0 & =\left.\frac{\mathrm{d}}{\mathrm{d} \epsilon}\right|_{\epsilon=0} M_{\epsilon}^{t} \cdot I \cdot M_{\epsilon}=\dot{M}_{0}^{t} \cdot I \cdot M_{0}+M_{0}^{t} \cdot I \cdot \dot{M}_{0} \\
& =\dot{M}_{0}^{t} \cdot I+I \cdot \dot{M}_{0} \\
& =\left(\begin{array}{cc}
\dot{A}_{0}^{t} & \dot{C}_{0}^{t} \\
\dot{B}_{0}^{t} & \dot{D}_{0}^{t}
\end{array}\right) \cdot\left(\begin{array}{cc}
0 & -\mathrm{Id} \\
\mathrm{Id} & 0
\end{array}\right)+\left(\begin{array}{cc}
0 & -\mathrm{Id} \\
\mathrm{Id} & 0
\end{array}\right) \cdot\left(\begin{array}{cc}
\dot{A}_{0} & \dot{B}_{0} \\
\dot{C}_{0} & \dot{D}_{0}
\end{array}\right) \\
& =\left(\begin{array}{cc}
\dot{C}_{0}^{t} & -\dot{A}_{0}^{t} \\
\dot{D}_{0}^{t} & -\dot{B}_{0}^{t}
\end{array}\right)+\left(\begin{array}{cc}
-\dot{C}_{0} & -\dot{D}_{0} \\
\dot{A}_{0} & \dot{B}_{0}
\end{array}\right) .
\end{aligned}
$$

From (57) we obtain

$$
\mathfrak{s p}_{2 n}=\left\{\dot{M}_{0}=\left(\begin{array}{cc}
-\dot{D}_{0}^{t} & \dot{B}_{0} \\
\dot{C}_{0} & \dot{D}_{0}
\end{array}\right) \mid \dot{B}_{0} \in S^{2} V, \dot{C}_{0} \in S^{2} V^{*}, \dot{D}_{0} \in \mathfrak{g l}(V)\right\}
$$

whence 56 become

$$
\dot{M}_{0} \cdot h=\dot{C}_{0}+\dot{D}_{0} \cdot h+h \cdot \dot{D}_{0}^{t}-h \cdot \dot{B}_{0} \cdot h .
$$


The decomposition (50) is implicitly written already in $(58)$, and it should be interpreted as a $|1|$-grading ${ }^{3}$ i.e.,

$$
\mathfrak{s p}_{2 n}=\underbrace{S^{2} V^{*}}_{\operatorname{deg}=-1} \oplus \underbrace{\mathfrak{g l}(V)}_{\operatorname{deg}=0} \oplus \underbrace{S^{2} V}_{\operatorname{deg}=+1} .
$$

In particular, it follows from 60 that both $S^{2} V^{*}$ and $S^{2} V$ are Abelian Lie algebras with a (natural) structure of $\mathfrak{g l}(V)$-module. Accordingly, the subgroup $P$ defined by 53 . corresponds infinitesimally to the non-negative part of the grading:

$$
\mathfrak{p}=\mathfrak{g l}(V) \oplus S^{2} V .
$$

The remaining part, that is $S^{2} V^{*}$, is canonically identified with the tangent space at $\arctan (0)$ to $\operatorname{LGr}(n, 2 n)$ :

$$
T_{\arctan (0)} \operatorname{LGr}(n, 2 n) \equiv S^{2} V^{*} .
$$

Identification $(62)$ will play a crucial role in the sequel. We stress here that, due to the presence of a quadratic term in $h$ in $(59)$, the isotropy action of $P$ on the tangent space $T_{\arctan (0)} \operatorname{LGr}(n, 2 n)$ reduces to the natural action of its 0 -graded part, that is $\mathfrak{g l}(V)$, on $S^{2} V^{*}$. The action of its 1 -graded part, that is $S^{2} V$, becomes visible only when the principal bundle

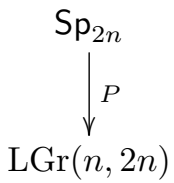

is identified with a sub-bundle of the second-order frame bundle of $\operatorname{LGr}(n, 2 n)$ (see Section 1.11 below).

1.10. The tautological and the tangent bundle of $\operatorname{LGr}(n, 2 n)$. We discuss now two important linear bundles that can be naturally associated with $\operatorname{LGr}(n, 2 n)$ - the tautological (rank- $n$ ) bundle and the tangent bundle (whose rank is $\frac{n(n+1)}{2}$ ). The key observation is that the latter can be identified with the second symmetric power of the dual of the former. Definitions can be easily given in terms of associated bundles to the $P$-principal bundle (63) introduced above. The key identification, on the other hand, is more evident from a local perspective.

From the $P$-principal bundle 63 one immediately obtains the linear bundle $\mathrm{Sp}_{2 n} \times{ }^{P} S^{2} V^{*}$ by letting $P$ act on $S^{2} V^{*}$ naturally through its 0 -graded part and trivially through the rest. By definition, the associated bundle is precisely the tangent bundle to $\operatorname{LGr}(n, 2 n)$, viz.

$$
T \operatorname{LGr}(n, 2 n)=\mathrm{Sp}_{2 n} \times{ }^{P} S^{2} V^{*} .
$$

We can regard (64) as a generalisation of 62 above, in the sense that the former, evaluated at $\arctan (0)$, gives the latter. The very identification (64) indicates also how

\footnotetext{
${ }^{3}$ See [11, Definition 3.1.2] for the general definition of a $|k|$-grading.
} 
to define a linear rank-n bundle, whose dualised symmetric square coincides with the tangent bundle to $\operatorname{LGr}(n, 2 n)$. It suffices to rewrite (64) as

$$
T \operatorname{LGr}(n, 2 n)=S^{2}\left(\mathrm{Sp}_{2 n} \times{ }^{P} V\right)^{*} .
$$

Indeed, at the right-hand side of (65), we see now the symmetric square of the dual of the following rank- $n$ bundle

$$
\begin{gathered}
L:=\operatorname{Sp}_{2 n} \times{ }^{P} V \\
\downarrow \\
\operatorname{LGr}(n, 2 n) .
\end{gathered}
$$

We call (66) the tautological bundle and we denote it by the symbol $L$. The choice of the letter $L$ is not accidental: if the same symbol $L$ denotes both the total space of the bundle (66) over $\operatorname{LGr}(n, 2 n)$ and a point $L \in \operatorname{LGr}(n, 2 n)$, then

$$
L_{L}=L
$$

that is, the fibre of $L$ at $L$ is again $L$-whence the modifier "tautological". With this notation, 65 becomes simply

$$
T \operatorname{LGr}(n, 2 n)=S^{2} L^{*}
$$

Observe that by

$$
T_{L} \operatorname{LGr}(n, 2 n)=S^{2} L^{*}
$$

we mean that the bundle identification 68 has been evaluated at the particular point $L \in \operatorname{LGr}(n, 2 n)$, thus becoming an identification of linear spaces. The reader should be aware that $(68)$ is an identification of bundles, whereas 69 is an identification of linear spaces, in spite of the usage of the same symbol $L$.

The importance of $(68)$ is that it allows us to speak about the rank of a tangent vector to $\operatorname{LGr}(n, 2 n)$, which is the rank of the corresponding bilinear form on the tautological bundle. In particular, rank-one vectors will be tightly connected to the key notion of a characteristic of a second-order PDE (see Sections 1.14 and 1.15.

For the reader feeling uncomfortable with the language of induced bundles we propose another explanation of the identification (69). Regard $L$ as a point of the Grassmannian $\operatorname{Gr}\left(n, V \oplus V^{*}\right)$ of $n$-dimensional subspaces of $V \oplus V^{*}$ (see (3p) and observe that the arctan map (4) can be generalised by choosing an arbitrary complement $L^{c}$ of $L$ in $V \oplus V^{*}$ and by defining

$$
\arctan _{L}: \operatorname{Hom}\left(L, L^{c}\right) \longrightarrow \operatorname{Gr}\left(n, V \oplus V^{*}\right)
$$

exactly the same way as arctan. Now the symplectic form $\omega$ allows us to identify $L^{c}$ with the dual $L^{*}$, whence $\operatorname{Hom}\left(L, L^{c}\right)$ with $L^{*} \otimes L^{*}$. The differential at 0 of 70 gives then an isomorphism between $L^{*} \otimes L^{*}$ and $T_{L} \operatorname{Gr}\left(n, V \oplus V^{*}\right)$, which one shows not to depend upon the choice of $L^{c}$. Finally, by similar reasonings as those in Section 1.2 one finds out that the subspace $S^{2} L^{*}$ corresponds precisely to the subspace $T_{L} \operatorname{LGr}(n, 2 n)$, thus obtaining 69 . 
1.11. The second-order frame bundle. For any $L \in \operatorname{LGr}(n, 2 n)$ we define the space 4 of second-order frames at $L$ as

$$
\mathcal{F}_{L}^{2}:=\left\{j_{0}^{2}(\phi) \mid \phi: S^{2} V^{*} \rightarrow \operatorname{LGr}(n, 2 n), \phi(0)=L, \phi \text { local diffeomorphism at } 0\right\},
$$

and the second-order frame bundle of $\operatorname{LGr}(n, 2 n)$ by

$$
\mathcal{F}^{2}:=\coprod_{L \in \operatorname{LGr}(n, 2 n)} \mathcal{F}_{L}^{2} .
$$

This bundle allows us to "see" the action of the positive-degree part of the group $P$. Recall that, by its definition (52), $P$ consists of diffeomorphisms of $\operatorname{LGr}(n, 2 n)$ preserving $\arctan (0)$. In particular, each $q \in P$ can be regarded as a local diffeomorphism $q: S^{2} V^{*} \rightarrow$ $\operatorname{LGr}(n, 2 n)$ sending 0 into $\arctan (0)$. Therefore, $j_{0}^{2}(q) \in \mathcal{F}_{\arctan (0)}^{2}$ and we found the map

$$
\begin{aligned}
& P \longrightarrow \mathcal{F}_{\arctan (0)}^{2}, \\
& q \longmapsto j_{0}^{2}(q) .
\end{aligned}
$$

Obviously, $\mathcal{F}_{\arctan (0)}^{2}$ is a group, and it can be proved that 73 above is a group embedding. Therefore, the structure group $P$ of the bundle $\operatorname{Sp}_{2 n} \rightarrow \operatorname{LGr}(n, 2 n)$ embeds into the structure group of the bundle $\mathcal{F}^{2} \rightarrow \operatorname{LGr}(n, 2 n)$. Then the $P$-principal bundle $\mathrm{Sp}_{2 n} \rightarrow \operatorname{LGr}(n, 2 n)$ can be regarded as a reduction of the second-order frame bundle of $\operatorname{LGr}(n, 2 n)$.

The reduction is easier grasped on the Lie algebra level. Indeed, the Lie algebra of the group $\mathcal{F}_{\arctan (0)}^{2}$ is

$$
\mathfrak{g l}\left(S^{2} V^{*}\right) \oplus\left(S^{2}\left(S^{2} V^{*}\right) \otimes S^{2} V\right)
$$

and it contains $\mathfrak{p}$ as a subalgebra. The embedding is indicated by $(59)$. The 0 -degree component of $\mathfrak{p}$ embeds naturally into $\mathfrak{g l}\left(S^{2} V^{*}\right)$. An element $B \in S^{2} V$, that is the 1-degree component of $\mathfrak{p}$, is mapped into the bilinear map

$$
\begin{gathered}
S^{2} V^{*} \times S^{2} V^{*} \longrightarrow S^{2} V, \\
(h, k) \longmapsto h \cdot B \cdot k .
\end{gathered}
$$

Regarding $\mathrm{Sp}_{2 n}$ as a sub-bundle of the second-order frame bundle of $\operatorname{LGr}(n, 2 n)$ is an indispensable step when it comes to the problem of equivalence of hypersurfaces in $\operatorname{LGr}(n, 2 n)$. Such a problem is usually dealt with, in the spirit of Cartan, via the moving frame methods, i.e., restrictions of $\mathcal{F}^{k}$ to the embedded hypersurfaces at hand.

1.12. Representation theory of $\mathrm{Sp}_{2 n}$ and its subgroup $\mathrm{GL}_{n}$. The standard choice of a Cartan subalgebra of $\mathfrak{s p}_{2 n}$ is given by the $n$-dimensional Abelian subalgebra

$$
\mathfrak{h}:=\left\langle\epsilon^{i} \otimes e_{i} \mid i=1, \ldots, n\right\rangle
$$

of diagonal matrices in $\mathfrak{g l}(V)$. The fundamental weights are then

$$
\lambda_{j}:=\sum_{i=1}^{j} e_{i} \otimes \epsilon^{i}, \quad j=1, \ldots, n
$$

\footnotetext{
${ }^{4}$ See, e.g., [27, Chapter IV], [26, Example 5.2], for more details on frame bundles.
} 
(see [11, Section 2.2.13]) where $e_{i} \otimes \epsilon^{i} \in \mathfrak{h}^{*}$ is the basis element dual to $\epsilon^{i} \otimes e_{i} \in \mathfrak{h}$. For any $j=1, \ldots, n$, the fundamental weight $\lambda_{j}$ appears as the weight of the highest weight vector

$$
v_{\lambda_{j}}:=e_{1} \wedge \ldots \wedge e_{j}
$$

in $\wedge^{j}\left(V \oplus V^{*}\right)$. Observe that

$$
\left[v_{\lambda_{n}}\right]=\operatorname{vol}(\arctan (0)),
$$

that is, the Plücker image of the origin $\arctan (0) \in \operatorname{LGr}(n, 2 n)$ is the line through the highest weight vector in $\wedge^{n}\left(V \oplus V^{*}\right)$. The subtle point is that $\wedge^{n}\left(V \oplus V^{*}\right)$ is not the highest weight module $V_{\lambda_{n}}$ of $v_{\lambda_{n}}$. Indeed, $\wedge^{n}\left(V \oplus V^{*}\right)$ is not irreducible and

$$
V_{\lambda_{n}}=\wedge_{0}^{n}\left(V \oplus V^{*}\right)
$$

is precisely the subspace introduced in $(23)$ above. Therefore, $\mathbb{P} V_{\lambda_{n}}$ is the representationtheoretic way of describing the Plücker embedding space.

Since $\mathrm{GL}(V) \subset \mathrm{Sp}\left(V \oplus V^{*}\right)$, any irreducible representation of $\operatorname{Sp}\left(V \oplus V^{*}\right)$ becomes a (not necessarily irreducible) representation of $\mathrm{GL}(V)$. In particular, the irreducible $\operatorname{Sp}\left(V \oplus V^{*}\right)$-representation $V_{\lambda}$ with highest weight $\lambda$ splits into several $\mathrm{GL}(V)$-irreducible representations. Only one of the latter contains the weight vector $v_{\lambda}$ and therefore it will be denoted by $L_{\lambda}$ :

$$
L_{\lambda}=\text { the unique } \mathrm{GL}(V) \text {-irreducible component of } V_{\lambda} \text { containing } v_{\lambda} \text {. }
$$

For instance, $V_{\lambda_{1}}$ is the $2 n$-dimensional fundamental representation $V \oplus V^{*}$, whereas $L_{\lambda_{1}}$ is simply $V$. Similarly, $V_{\lambda_{n}}$ is the (very large) de-projectivised Plücker embedding space for $\operatorname{LGr}(2 n, n)$, whereas $L_{\lambda_{n}}$ is the one-dimensional line $\operatorname{vol}(\arctan (0))$ !

1.13. The tautological line bundle and $r$-th degree hypersurface sections. Having at one's disposal the $P$-principal bundle 63 and regarding the $\mathrm{GL}_{n}$-irreducible representation $L_{\lambda}$ as a representation of $P$, one can form the associated vector bundle

$$
\mathrm{Sp}_{2 n} \times{ }^{P} L_{\lambda} .
$$

For instance, with $\lambda=\lambda_{1}$ one obtains the tautological bundle introduced earlier (cf. (66), (67) ) and with $\lambda=\lambda_{n}$ one obtain the tautological line bundle $\mathcal{O}_{\operatorname{LGr}(n, 2 n)}(-1)$. The readear should bear in mind that the former is a rank- $n$ bundle, whereas the latter has rank 1. Indeed, there are two "tautological" principles at play here, following from the fact that $\operatorname{LGr}(n, 2 n)$ is made of $n$-dimensional linear subspaces, and $\mathbb{P} V_{\lambda_{n}}$ consists of lines, respectively.

By definition, $\mathcal{O}_{\operatorname{LGr}(n, 2 n)}(-1)$ is the pull-back via the Plücker embedding of the tautological line bundle over the Plücker embedding space $\mathbb{P} V_{\lambda_{n}}$. Indeed, the fibre of $\mathcal{O}_{\operatorname{LGr}(n, 2 n)}(-1)$ at $L \in \operatorname{LGr}(n, 2 n)$ is $\operatorname{vol}(L)$ itself, understood not as a point of $\mathbb{P} V_{\lambda_{n}}$, but as an abstract one-dimensional linear space. Such is the standard notation of Algebraic Geometry: over the projective space $\mathbb{P}(W)$ one has a group (isomorphic to $\mathbb{Z}$ ) of linear bundles

$$
\mathcal{O}(-1)_{[w]}:=\langle w\rangle, \quad \mathcal{O}(1):=\mathcal{O}(-1)^{*}, \quad \mathcal{O}( \pm r):=\mathcal{O}( \pm 1)^{\otimes r}, \quad \forall[w] \in \mathbb{P} W, r \in \mathbb{N} .
$$


Given a hyperplane $\pi \in \mathbb{P} V_{\lambda_{n}}^{*}$, we may form the hyperplane section $\Sigma_{\pi}:=\pi \cap \operatorname{LGr}(n, 2 n)$. The same hypersurface $\Sigma_{\pi}$ can be described as the zero locus of a suitable section of $\mathcal{O}_{\operatorname{LGr}(n, 2 n)}(1)$, the dual of $\mathcal{O}_{\operatorname{LGr}(n, 2 n)}(-1)$. Indeed, let $\alpha \in V_{\lambda_{n}}^{*}$ be a linear form such that $\pi=\mathbb{P}(\operatorname{ker} \alpha)$. Then $\alpha$ can be restricted to each line $\operatorname{vol}(L)$, thus yielding a section (still denoted by $\alpha$ ) of $\mathcal{O}_{\operatorname{LGr}(n, 2 n)}(1)$. The value of the section $\alpha$ at $L$ is simply $\left.\alpha\right|_{\operatorname{vol}(L)}$. Therefore, the zero locus of $\alpha$ is made precisely by those $L$, such that $\operatorname{vol}(L) \subset \operatorname{ker} \alpha$, that is, $\operatorname{vol}(L) \in \pi$, which is precisely $\Sigma_{\pi}$.

A central question in the geometry of PDEs is: how to recognise a hyperplane section? In the above language of induced bundles, this is the same as asking: when a section of $\mathcal{O}_{\mathrm{LGr}(n, 2 n)}(1)$ comes from a linear form $\alpha \in V_{\lambda_{n}}^{*}$ ?

In general, the map

$$
S^{r} V_{\lambda_{n}}^{*} \longrightarrow \Gamma\left(\operatorname{LGr}(n, 2 n), \mathcal{O}_{\operatorname{LGr}(n, 2 n)}(r)\right)
$$

associating with a degree- $r$ homogeneous polynomial on $V_{\lambda_{n}}$ a (global) section of $\mathcal{O}_{\operatorname{LGr}(n, 2 n)}(r)$, the $r$-th power of $\mathcal{O}_{\operatorname{LGr}(n, 2 n)}(1)$, can be resolved. More precisely, there exists a differential operator $\square^{r}$ acting on sections of $\mathcal{O}_{\operatorname{LGr}(n, 2 n)}(r)$, whose kernel is precisely the image of (81). The construction of $\square^{r}$ is by no means trivial and it is based on the so-called BGG resolution [22, Theorem 5.9].

From the point of view of PDEs, the operator $\square^{r}$ is to be understood as a test, that is, as a criterium to establish whether a given second-order PDE $F\left(p_{i j}\right)=0$ belongs to the well-defined ( $\mathrm{Sp}_{2 n}$-invariant) class of $r$-th degree hypersurface sections. Running the test entails applying $\square^{r}$ to the function $F$ defining the equation at hand. Therefore, the above class of second-order PDEs is to be understood as the set of solutions of the special differential equation $\square^{r}(F)=0$. The same idea will be applied to another important class of PDEs, the integrable ones, see Section 2.8 below.

1.14. Rank-one vectors. An immediate consequence of the fundamental isomorphism (68) is that the projectivized tangent bundle $\mathbb{P} T \operatorname{LGr}(n, 2 n)$ contains a proper sub-bundle, namely the bundle

$$
\mathcal{R}:=\{[v] \in \mathbb{P} T \operatorname{LGr}(n, 2 n) \mid \operatorname{rank} v=1\}
$$

of (projective classes of) rank-one vectors. Indeed, (68) allows us to speak of the rank of the vector $v$, and such a notion is well-defined and depends only on the projective class of $v$.

We provide now an interesting characterisation of rank-one tangent vectors. Let $L \in$ $\operatorname{LGr}(n, 2 n)$ be an arbitrary point, and $v$ a tangent vector at $L$ to $\operatorname{LGr}(n, 2 n)$. Let $\gamma=\gamma(\epsilon)$ be a curve passing through $L$ with speed $v$. Then, each point $\gamma(\epsilon)$ can be interpreted as a Lagrangian subspace of $V \oplus V^{*}$, and in particular $\gamma(0)=L$. Observe that, even for small values of $\epsilon$, the intersection $\gamma(\epsilon) \cap \gamma(0)$ needs not to be nontrivial.

Here it comes the peculiarity of rank-one vectors: $v$ is rank-one if and only if the curve $\gamma$ can be chosen in such a way as the intersection $\gamma(0) \cap \gamma(\epsilon)$ is a fixed hyperplane $\Sigma \subset L$ (that is, not depending on $\epsilon$ ) for all $\epsilon$ in a small neighbourhood of zero. In other words, there is a correspondence between rank-one tangent vectors at $L$ and hyperplanes $\Sigma \subset L$, that is, elements of $\mathbb{P} L^{*}$. In one direction, such a correspondence is quite evident. 
Thanks to the homogeneity of $\operatorname{LGr}(n, 2 n)$ we can work at the origin $\arctan (0)$ (see Section 1.9 above). Let $[\alpha] \in \mathbb{P} \arctan (0)^{*}$ represent the hyperplane $\Sigma_{\alpha}:=\operatorname{ker} \alpha$. We need to describe the generic Lagrangian plane $L$, which is "close" to $\arctan (0)$, and intersects the latter precisely along $\Sigma_{\alpha}$. Since $L$ has to be "close" to $\arctan (0)$, we can assume it to belong to the big cell, that is, to be of the form $\arctan (h)$, for some $h \in S^{2} V^{*}$. The key remark, rather obvious, is that

$$
\arctan (0) \cap \arctan (h)=\operatorname{ker} h .
$$

So, the above intersection coincides with $\Sigma_{\alpha}$ if and only if ker $\alpha=\operatorname{ker} h$. That is, if and only if the quadratic form $h$ is proportional to the square of the linear form $\alpha$. Therefore, the correspondence between hyperplanes in $L$ and rank-one tangent vectors to $\operatorname{LGr}(n, 2 n)$ at $L$ is nothing but

$$
\begin{gathered}
\mathbb{P} L^{*} \longrightarrow \mathbb{P} S^{2} L^{*} \\
{[\alpha] \longmapsto\left[\alpha^{2}\right],}
\end{gathered}
$$

one of the most fundamental maps in classical Algebraic Geometry: the Veronese embedding [23. Example 2.4]. The above map (84), in the context of second order PDEs, allows us to establish an important relationship between objects depending on second order derivatives (elements of $S^{2} L^{*}$ are reminiscent of Hessian matrices) and objects depending on first order derivatives (elements of $L^{*}$ correspond to covectors on the space of independent variables). This point of view will be clarified in Section 2.6

1.15. Characteristics. In compliance with the terminology found, e.g., in [2, Formula (3.1)], [8, Chapter VI] and [T3, Part II], we denote by

$$
\Sigma^{(1)}:=\{L \in \operatorname{LGr}(n, 2 n) \mid L \supset \Sigma\}
$$

the prolongation of the hyperplane $\Sigma \in \mathbb{P} L^{*}$. As we have already pointed out, $\Sigma^{(1)}$ is a line passing through $L$ itself (see Section 1.14). In fact, via Plücker embedding, $\Sigma^{(1)}$ becomes an actual projective line in $\mathbb{P} V_{\lambda_{n}}$, see, e.g., [2, Proposition 2.1].

Moreover, if $\Sigma=\operatorname{ker} \alpha$, with $\alpha \in L^{*}$, then

$$
T_{L} \Sigma^{(1)}=\left\langle\alpha^{2}\right\rangle
$$

as a subset of $S^{2} L^{*}=T_{L} \operatorname{LGr}(n, 2 n)$ (recall formula 69$)$ ). Let $\mathcal{E} \subseteq \operatorname{LGr}(n, 2 n)$ be a submanifold and $L \in \mathcal{E}$. Then $\Sigma$ is called a characteristic (resp., strong characteristic) for $\mathcal{E}$ at $L$ if $\Sigma^{(1)}$ is tangent to (resp., contained in) $\mathcal{E}$. These notions will be essential in the analysis of the well-posedness of initial value problems for PDEs, see Section 2.4

1.16. The Lagrangian Chow transform. So far we have worked with Lagrangiani.e., maximally isotropic - subspaces $L$ of $V \oplus V^{*}$. The hyperplanes $\Sigma$ appearing in Section 1.15 are the first instances of sub-maximal isotropic subspaces (in this case, $(n-1)$ dimensional). In fact, nothing forbids considering the sets

$$
\operatorname{LGr}\left(i, V \oplus V^{*}\right):=\left\{L \in \operatorname{Gr}\left(i, V \oplus V^{*}\right)|\omega|_{L} \equiv 0\right\}, \quad i=1, \ldots, n,
$$

which we may call "Lagrangianoid Grassmannians", as well as the corresponding incidence correspondences:

$$
\mathrm{Fl}^{\text {iso }}\left(V \oplus V^{*} ; i, j\right):=\left\{\left(L_{1}, L_{2}\right) \in \operatorname{LGr}\left(i, V \oplus V^{*}\right) \times \operatorname{LGr}\left(j, V \oplus V^{*}\right) \mid L_{1} \subset L_{2}\right\},
$$

where $1 \leq i<j \leq n$. 
In particular, an important role is played by $\operatorname{LGr}\left(1, V \oplus V^{*}\right) \equiv \mathbb{P}\left(V \oplus V^{*}\right)$ and $\mathrm{LGr}\left(n-1, V \oplus V^{*}\right)$. Indeed, in the geometric theory of PDEs, the former describes rankone subdistributions of the contact distribution, and the latter describes infinitesimal Cauchy data. The two notions coincide for $n=2 !^{5}$

A classical observation in Algebraic Geometry is that all these "Lagrangianoid Grassmannians" are tied together by means of the incidence correspondences (88). Indeed, the above-defined sets of isotropic flags fit into the following double fibration:

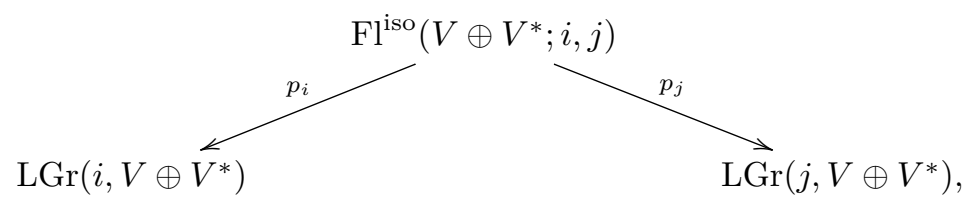

with $i<j$. For instance, with $i=n-1$ and $j=n$ diagram 89 reads

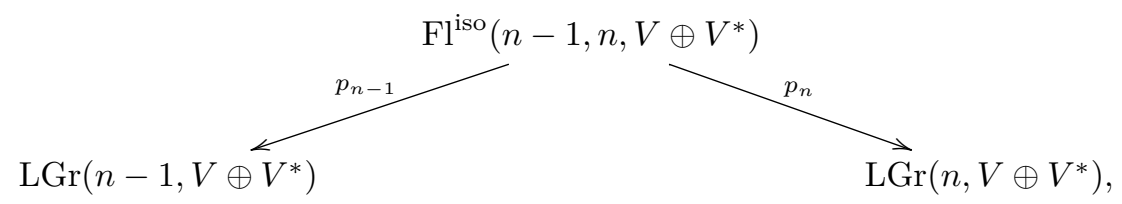

and for any $\Sigma \in \operatorname{LGr}\left(n-1, V \oplus V^{*}\right)$, the "double fibration transform" $p_{n}\left(p_{n-1}^{-1}(\Sigma)\right)$ of $\Sigma$ is precisely the prolongation $\Sigma^{(1)}$ defined by $(85)$. Conversely, for any $L \in \operatorname{LGr}\left(n, V \oplus V^{*}\right)$, the "inverse double fibration transform" $p_{n-1}\left(p_{n}^{-1}(L)\right)$ of $L$ is nothing but $\mathbb{P} L^{*}$.

Another interesting example is obtained with $i=1$ and $j=n$. Diagram 89 then reads

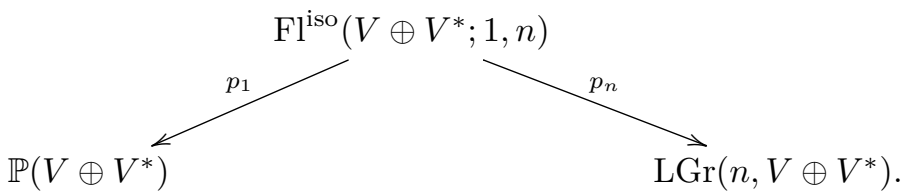

The above diagram allows us to recast a simple but useful theorem, known in Algebraic Geometry as the Chow form/transform: if $X \subset \mathbb{P}\left(V \oplus V^{*}\right)$ is a smooth variety of dimension $n-1$, then its "double fibration transform" $p_{n}\left(p_{1}^{-1}(X)\right)$ is a smooth hypersurface in $\operatorname{LGr}\left(n, V \oplus V^{*}\right)$, of the same degree as $X$ [3, Lemma 23]. The latter will be referred to as the Lagrangian Chow transform of $X$. We stress that the notion of degree in $\operatorname{LGr}\left(n, V \oplus V^{*}\right)$ refers to the surrounding Plücker embedding space.

As a nice example consider an $n$-dimensional (not necessarily Lagrangian) subspace $D \subset V \oplus V^{*}$. Then $\mathbb{P} D$ is a (smooth) $(n-1)$-dimensional variety in $\mathbb{P}\left(V \oplus V^{*}\right)$ whose Lagrangian Chow transform reads

$$
\operatorname{det}(D-h)=0,
$$

where $D$ is the (not necessarily symmetric) $n \times n$ matrix corresponding to the subspace $D$ in the big cell $V^{*} \otimes V^{*}$ of $\operatorname{Gr}\left(n, V \oplus V^{*}\right)$ and $h$ is the symmetric $n \times n$ matrix corresponding

\footnotetext{
${ }^{5}$ The classical reference in the book 37]. Different treatments of the subject, sometimes closer in spirit to the present paper, can be found, e.g., in [46, 34, 2].
} 
to the generic element of the big cell $S^{2} V^{*}$ of $\operatorname{LGr}\left(n, V \oplus V^{*}\right)$. Observe that 92 , though containing all the minors of $h$, is linear in the Plücker coordinates, as predicted by the theorem.

The second order PDEs corresponding to the Lagrangian Chow transforms of the $n$-dimensional sub-distributions of the contact distribution are the so-called Goursat-type Monge-Ampère equations, introduced by E. Goursat in 1899 [21], way before the inception of the Chow transform, see Section 2.6 below. It is precisely thanks to the introduction of the Lagrangian Chow transform that the notion of a Goursat-type Monge-Ampère equation can be generalised to arbitrary conic sub-distributions of the contact distribution [T5, Section 9].

1.17. A few remarks on $\operatorname{LGr}(2,4)$ and $\operatorname{LGr}(3,6)$. We conclude this survey of the rich geometry of $\operatorname{LGr}(n, 2 n)$ by pointing out the peculiarities of two low-dimensional examples, namely when $n=2$ or $n=3$. The case $n=2$ is examined from top to bottom in the paper [43. Even if the case $n=3$ does not boast its own treatise, the reader will find specific facts and results in [T4, Section 5] and [22, Section 4.2]. We do not review here all that can be found in the aforementioned works - we rather highlight the origin of the interestingness and diversity of these two cases.

The departing point is the fact, already pointed out, that $\operatorname{LGr}(n, 2 n)$ is always isotropic with respect to the natural two-form defined on the (de-projectivised) Plücker embedding space $V_{\lambda_{n}}$, see 79 .

In the case $n=2$, this two-form is symmetric (see (37) ) and we denote it here by $g$. Therefore, since the codimension of $\operatorname{LGr}(2,4)$ in $\mathbb{P} V_{\lambda_{2}} \equiv \mathbb{P}^{4}$ is one, $\operatorname{LGr}(2,4)$ must coincide with the (projectivised) null cone of $g$ in $\mathbb{P}^{4}$.

In the real case, $g$ has signature $(+++--)$ and then $\operatorname{LGr}(2,4)$ inherits a conformal structure of signature $(++-)$. Such an $\mathrm{Sp}_{4}(\mathbb{R})$-invariant conformal structure is precisely the one that has been used by The to carry out a classification of hypersurfaces in $\operatorname{LGr}(2,4)$ by the method of moving frames [43]. The same structure has also been used by the authors to characterise the hyperplane sections of $\operatorname{LGr}(2,4)$ in terms of the trace-free second fundamental form [22, Corollary 4.2].

Another peculiarity of $\operatorname{LGr}(2,4)$ which is worth recalling is that $\operatorname{LGr}(2,4)$ is isomorphic to the so-called Lie quadric. This is the moduli space of all circles in $\mathbb{R}^{2}$, i.e., including also those with zero or infinite radius. Such an isomorphism was essentially known to S. Lie himself [42, Lie's Memoir on a Class of Geometric Transformations, Section 9], though it can be rephrased in modern language by using Hopf fibration, see [5. Section 5] and [6].

Passing to the case $n=3$, we see that the 6 -dimensional $\operatorname{LGr}(3,6)$ does not carry any natural conformal structure in the usual sense. Nevertheless a "trivalent" analogue of a conformal structure can still be defined on $\operatorname{LGr}(3,6)$. Such a structure has been exploited by the authors to characterise hyperplane sections of $\operatorname{LGr}(3,6)$ in terms of a suitable generalisation of the trace-free second fundamental used in the case $n=2[22$, Section 4.2].

Another really intriguing feature of $\operatorname{LGr}(3,6)$, or rather of its Plücker embedding in $\mathbb{P}^{13}$, is that such an embedding can be regarded as an appropriate generalisation of 
the twisted cubic in $\mathbb{P}^{3}$, whereby the field of complex number has been replaced by the Jordan algebra of symmetric $3 \times 3$ matrix. This analogy played a fundamental role in a recent analysis of PDEs with prescribed group of symmetries 44. A gentle introduction to it can be found in [T4, Section 5].

\section{Hypersurfaces in the (real) Lagrangian Grassmannian and second order}

PDEs. In the second part of this paper we examine more in depth the geometry of hypersurfaces in the Lagrangian Grassmannian $\operatorname{LGr}(n, 2 n)$. Some of the key notions, like those of a hyperplane section (Section 1.7), of an $r$-th degree section (Section 1.13) and of the characteristic of a hypersurface (Section 1.15), have already been introduced above. It was also anticipated that these ideas were going to have interesting incarnations in the context of second order PDEs. All of this will be explained below.

From now on, we work in the real smooth category.

2.1. Contact manifolds and second order PDEs. The idea of framing second order PDEs against the general background of contact manifolds and their prolongations is rather old and, in a sense, it belongs to the mathematical folklore. An excellent treatise of this topic is the book [30] though a slenderer introduction can be found in [17].

The departing point is a contact manifold $(M, \mathcal{C})$, that is a $(2 n+1)$-dimensional smooth manifold equipped with a one-codimensional distribution $\mathcal{C}$, such that the Levi form,

$$
\begin{gathered}
\omega: \mathcal{C} \wedge \mathcal{C} \longrightarrow T M / \mathcal{C}, \\
(X, Y) \longmapsto[X, Y]+\mathcal{C},
\end{gathered}
$$

is non-degenerate. The so-called Darboux coordinate may help to clarify the picture: $M$ is (locally) described by the coordinates

$$
\left(x^{1}, \ldots, x^{n}, u, p_{1}, \ldots, p_{n}\right),
$$

the distribution $\mathcal{C}$ is (locally) spanned by the $2 n$ vector fields

$$
\underbrace{D_{1}:=\partial_{x^{1}}+p_{1} \partial_{u}, \ldots, D_{n}:=\partial_{x^{n}}+p_{n} \partial_{u}}_{\text {"total" derivatives }}, \underbrace{\partial_{p_{1}}, \ldots, \partial_{p_{n}}}_{\text {"vertical" derivatives }}
$$

and (locally)

$$
\omega=d x^{i} \wedge d p_{i} .
$$

The next step consists in regarding each contact plane $\mathcal{C}_{p}$, with $p \in M$, as a symplectic linear space (thanks to the symplectic form $\omega_{p}$ ) and in constructing the corresponding Lagrangian $\operatorname{Grassmannian} \operatorname{LGr}\left(\mathcal{C}_{p}\right):=\operatorname{LGr}\left(n, \mathcal{C}_{p}\right)$. One readily verifies that the total derivatives and the vertical derivatives are dual to each other via $\omega$, that is, they can be identified with the vectors $e_{i}$ and the covectors $\epsilon^{i}$ introduced in Section 1.1 respectively. Then, following the same procedure as in Section 1.2 we obtain coordinates $p_{i j}$ on $\operatorname{LGr}\left(\mathcal{C}_{p}\right)$. Doing the same for any point $p$ one obtains a bundle

$$
\operatorname{LGr}(\mathcal{C}):=\coprod_{p \in M} \operatorname{LGr}\left(\mathcal{C}_{p}\right) \longrightarrow M
$$


with fibre coordinates $p_{i j}$, known as the Lagrangian Grassmannian bundle of $M$ or the first prolongation of $M$ and sometimes denoted by $M^{(1)}$.

Then a hypersurface $\mathcal{E} \subset M^{(1)}$, being locally represented as

$$
\mathcal{E}: F\left(x^{1}, \ldots, x^{n}, u, p_{1}, \ldots, p_{n}, \ldots, p_{i j}, \ldots\right)=0,
$$

clearly corresponds to a second order PDE. Perhaps it is less evident that a solution of $\mathcal{E}$ is captured by a Lagrangian submanifold $L \subset M$, such that its tangent lift $T L$ is contained into $\mathcal{E}$. In the coordinates 94 of Darboux, $L=L_{f}:=\left\{\boldsymbol{x}, f(\boldsymbol{x}), \ldots,\left(\partial_{x^{i}} f\right)(\boldsymbol{x}), \ldots\right\}$, where $f$ is a function of $\boldsymbol{x}=\left(x^{1}, \ldots, x^{n}\right)$, and it is not hard to prove that $T L$ (the set of all the tangent $n$-dimensional subspaces to $L$ ) coincides with

$$
L_{f}^{(1)}:=\left\{\boldsymbol{x}, f(\boldsymbol{x}), \ldots,\left(\partial_{x^{i}} f\right)(\boldsymbol{x}), \ldots, \ldots,\left(\partial_{x^{i}} \partial_{x^{j}} f\right)(\boldsymbol{x}), \ldots\right\} \subset M^{(1)},
$$

so that $L_{f}^{(1)} \subset \mathcal{E}$ if and only if the function $f$ fulfills the (familiar looking) PDE appearing in 97 .

From now on we make the (non-restrictive) assumption that $\mathcal{E}$ is actually a sub-bundle of $M^{(1)}$. Then the fibres $\mathcal{E}_{p}$ of $\mathcal{E}$ are hypersurfaces in the corresponding Lagrangian Grassmannians $\operatorname{LGr}\left(\mathcal{C}_{p}\right)$, with $p \in M$. So, we are in position of utilising the theoretical machinery developed in the first part. Essentially, we are going to work with a family of symplectic spaces, Lagrangian Grassmannians and hypersurfaces of the latter, rather than with a fixed one. Besides the appearance of a fancy index " $p$ ", the techniques remain unchanged.

A subtler point, which may have escaped the hasty reader, is that passing from the point-wise perspective ("microlocal", as some love to say) to the global framework, the equivalence group has changed from the finite-dimensional Lie group $\mathrm{Sp}_{2 n}$ to the infinitedimensional contact group $\operatorname{Cont}(M)$.

2.2. Nondegenerate second order PDEs and their symbols. If one's ultimate goal is to be able to setup the equivalence problem for second order PDEs, then there is one rough distinction that can be made from the very beginning.

A hypersurface $\mathcal{E} \subset \operatorname{LGr}(n, 2 n)$ is called non-degenerate at $L$ if the tangent hyperplane $T_{L} \mathcal{E}$, understood as a line in $S^{2} L$ via the dual of identification 68 is made of nondegenerate elements. Then $\mathcal{E}$ is called non-degenerate if it is non-degenerate at all points. Finally, a second order PDE $\mathcal{E} \subset M^{(1)}$ is non-degenerate if so are all its fibres. Obviously, the property of being non-degenerate is $\operatorname{Cont}(M)$-invariant and hence defines a wellbehaved class of second order PDEs.

The fundamental correspondence (68) reads now, in terms of the local Darboux coordinates 94 ,

$$
\begin{gathered}
S^{2} L^{*} \longrightarrow T_{L} \operatorname{LGr}(n, 2 n), \\
\left.\mathrm{d} x^{i} \odot \mathrm{d} x^{j} \longmapsto \partial_{p_{i j}}\right|_{L} .
\end{gathered}
$$

Therefore, if $\mathcal{E}=\{F=0\}$ is a hypersurface in $\operatorname{LGr}(n, 2 n)$, then $\mathrm{d}_{L} F$ can be regarded as an element of $S^{2} L$, viz.

$$
\mathrm{d}_{L} F=\left.\left.\frac{\partial F}{\partial p_{i j}}\right|_{L} \mathrm{~d} p_{i j} \longleftrightarrow \frac{\partial F}{\partial p_{i j}}\right|_{L} \partial_{x^{i}} \odot \partial_{x^{j}}
$$


The symmetric rank-two contravariant tensor appearing at the right-hand side of 100 is of paramount importance in the theory of PDEs. It is called the symbol of $F$ at $L$. If the dependence upon $L$ is discarded then one has a section of the bundle $\left.S^{2} L\right|_{\mathcal{E}}$ (beware of the syncretism of the symbol $L$, cf. (68) and (69)), still called the symbol of $F$. Finally, if $\mathcal{E} \subset M^{(1)}$ is a second order PDE, then the symbol of $F$ must be understood as a section of a bundle over $\mathcal{E}$, whose restriction to the fibre $\mathcal{E}_{p}$ is the aforementioned bundle $\left.S^{2} L\right|_{\mathcal{E}_{p}}$. Such a proliferation of "bundles upon bundles" is a congenital feat of the theory and the reader must cope with it, see also [T3, Section 3]. Using the same symbol for the various incarnations of the same concept, far from bringing in more confusion, is the only way to keep the notation bearable.

Now we must face a fundamental problem in the theory of hypersurfaces, that is the fact that $F$ is not, of course, uniquely determined by $\mathcal{E}$ and it is $\mathcal{E}$ that we wish to study, not $F$. Usually things are simpler with $F$, but then one has to ensure the result to be independent upon the choic ${ }^{6}$ of $F$ in the ideal of $\mathcal{E}$. Another way out is to prove results directly on $\mathcal{E}$, but this usually demands a deeper abstraction.

For instance, the symbol of the equation $\mathcal{E}$ at $L \in M^{(1)}$ is the element

$$
\operatorname{Smbl}_{L}(\mathcal{E}):=\left[\mathrm{d}_{L} F\right] \in \mathbb{P}\left(S^{2} L\right),
$$

whereas the previously defined symbol of $F$ at $L$ is just a representative of it. One is more conceptual, the other more treatable. Nevertheless, both allow us to rephrase the notion of non-degeneracy: the $\operatorname{PDE} \mathcal{E}$ is non-degenerate at $L$ if its symbol at $L$ is a generic element of $\mathbb{P}\left(S^{2} L\right)$ or, equivalently, if the symbol of any representative $F$ of $\mathcal{E}$ is a non-degenerate rank-two symmetric tensor on $L$.

2.3. Symplectic second order PDEs. The various versions of the above notion of non-degeneracy (in a point, in a fibre, everywhere) stressed the main issue of passing from the study of hypersurfaces in $\operatorname{LGr}(n, 2 n)$ to the study of second order PDEs $\mathcal{E} \subset M^{(1)}$ : the fibres of $\mathcal{E}$ may fulfill some special property (e.g., that of being non-degenerate) over some subset $U \subset M$ and, simultaneously, may not fulfill it over $M \backslash U$. This is the main source of additional difficulties: two equations of "mixed type" may not be Cont $(M)$ equivalent for topological reasons (e.g., because the locus $U$ of the first equation is not homeomorphic to the analogous locus of the second equation).

A reasonable compromise between the $\mathrm{Sp}_{2 n}$-equivalence problem and the $\operatorname{Cont}(M)$ equivalence problem is provided by the sub-class of second order PDEs that locally look like

$$
\mathcal{E}: F\left(\ldots, p_{i j}, \ldots\right)=0,
$$

that is, exactly like (97), but without explicit dependency upon $x^{1}, \ldots, x^{n}, u, p_{1}, \ldots, p_{n}$. Such a class will be called the class of symplectic second order PDEs in compliance with the terminology adopted, e.g., in [16, 41, 15].7 More geometrically, one can speak about symplectic second order PDEs when the bundle $M^{(1)} \longrightarrow M$ is trivial, i.e.,

\footnotetext{
${ }^{6}$ Borrowing a terminology from Algebraic Geometry, we call the ideal of $\mathcal{E}$ the ideal in $C^{\infty}\left(M^{(1)}\right)$ of functions vanishing on $\mathcal{E}$.

${ }^{7}$ According to another school, this is the class of Hirota-type second order PDEs, see e.g., [18, 19].
} 
$M^{(1)}=M \times \operatorname{LGr}(n, 2 n)$ and $\mathcal{E}$ is the pull-back of a hypersurface (still denoted by $\mathcal{E}$ ) in $\operatorname{LGr}(n, 2 n)$. Hence, the modifier "symplectic" alludes to the fact that the equivalence group is still $\mathrm{Sp}_{2 n}$, even though the equation is defined over the contact manifold $M$. From now on, unless otherwise stated, all second order PDEs are assumed to be (everywhere) non-degenerate and symplectic. The same symbol $\mathcal{E}$ will be used both for the sub-bundle of $M^{(1)}$ and for an its generic fibre. The context will help the reader to know which is which.

It may happen that very hard questions for general second order PDEs become almost trivial in the context of symplectic second order PDEs. For instance, the problem of linearisability of a general parabolic Monge-Ampère equation, up to contactomorphisms, was raised by R. Bryant [9] and to date it is still open, whereas its analogue for symplectic Monge-Ampère equations is (relatively) trivial, see [T4, Theorem 1.4]. Obviously, the class of symplectic second order PDEs is not $\operatorname{Cont}(M)$-invariant.

2.4. The characteristic variety. Before introducing the simplest yet nontrivial class of PDEs, we recast the notion of a characteristic in the present context of PDEs. Recall that, for any point $L \in \mathcal{E}$, a hyperplane $\Sigma \in \mathbb{P} L^{*}$ is called a characteristic (resp., strong characteristic) for $\mathcal{E}$ at $L$ if the rank-one line $\Sigma^{(1)} \subset T_{L} \operatorname{LGr}(n, 2 n)$ it tangent to $\mathcal{E}$ at $L$ (resp., contained into $\mathcal{E}$ ), see Section 1.15 Let now $\mathcal{E} \subset M^{(1)}$ be a PDE, and $L \in \mathcal{E}$. The subset

$$
\Xi_{L}(\mathcal{E}):=\left\{\Sigma \in \mathbb{P} L^{*} \mid \Sigma^{(1)} \text { is tangent to } \mathcal{E} \text { at } L\right\} \subset \mathbb{P} L^{*}
$$

is called the characteristic variety of $\mathcal{E}$ at $L$. Their (disjoint) union, for all $L \in \mathcal{E}$, forms a bundle over $\mathcal{E}$ called simply the characteristic variety and denoted by $\Xi(\mathcal{E})$.

The conceptual definition (103 may be abstruse, but Darboux coordinates make it easily accessible to computations. It is easy to see that 103 can be equivalently formulated as

$$
\Xi_{L}(\mathcal{E})=\left\{[\alpha] \mid \alpha \in L^{*}, \operatorname{Smbl}_{L}(F)\left(\alpha^{2}\right)=0\right\} .
$$

Here $\operatorname{Smbl}_{L}(F)$ is the symbol of $F$ at $L$, as in the right-hand side of 100 . Observe that the condition at the right-hand side of 104 is independent upon the choice of $F$ in the ideal of $\mathcal{E}$.

In this section we merely provide the definition of the characteristic variety $\Xi(\mathcal{E})$. A careful examination of all the properties of $\Xi(\mathcal{E})$ and ramifications would fill a separate treatise. For more information, we refer the reader to [T3] in this very volume and to [46] and references therein. We just make two final remarks.

First, the characteristic variety $\Xi(\mathcal{E})$ can be used to carry out a rough classification of PDEs. For instance, $\mathcal{E}$ is non-degenerate at $L$ iff $\Xi_{L}(\mathcal{E})$ is a non-degenerat $8^{8}$ quadric. Similarly, $\mathcal{E}$ is elliptic at $L$ iff $\Xi_{L}(\mathcal{E})$ is empty. In the case $n=2, \mathcal{E}$ is parabolic at $L$ iff $\Xi_{L}(\mathcal{E})$ consists of two lines. And this list of examples may continue.

Second, the characteristic variety $\Xi(\mathcal{E})$ plays a fundamental role in the initial value problem. Assume, to make things even simpler, that a characteristic $\Sigma$ is strong. Then the entire line $\Sigma^{(1)}$ is contained into $\mathcal{E}$. This means that there is a family, parametrised by $\mathbb{P}^{1}$, of infinitesimal solutions to $\mathcal{E}$ admitting the same initial (infinitesimal) datum $\Sigma$.

\footnotetext{
${ }^{8}$ Beware that non-degenerate does not mean non-irreducible.
} 
In other words, if the initial datum is tangent to $\Sigma$ (in which case the initial datum is called characteristic), then the Cauchy-Kowalewskaya theorem fails in uniqueness. More examples clarifying this property of $\Xi(\mathcal{E})$ can be found in the above-cited paper [46].

2.5. Hyperplane sections and PDEs of Monge-Ampère type. In the literature, the Monge-Ampère equation is usually understood to be

$$
\operatorname{det}\left(p_{i j}\right)=f\left(x^{i}, u, p_{i}\right) .
$$

It is at the very heart of a feverish research activity: for instance, the book [45], concerning the problem of the optimal mass transportation, gathered almost one thousand citations in a dozen of years. Besides countless scientific and technological applications, the problem of optimal mass transportation can be formulated in important economical models, in the form of optimal allocation of resources. This led, among many other things, to a Nobel prize in the economic sciences for Kantorovich [25, 24, 1, 17].

On a more speculative level, one can ask for which functions $f$ in 105 one obtains a (Cont $(M)$-invariant) class of PDEs. For instance, there exists a family of functions $f$ such that the corresponding equation 105 can be brought into the linear form?

$$
p_{11}=0,
$$

by means of a (partial) Legendre transformation (that is a particular element of $\operatorname{Cont}(M))$, see, e.g., [17, 2]. This is the easiest example of a $\operatorname{Cont}(M)$-invariant subclass of Monge-Ampère equations - those having an integrable characteristic distribution. A linear (symplectic) second order PDE

$$
\mathcal{E}: F\left(p_{i j}\right)=B^{i j} p_{i j}=0, \quad B^{i j} \in \mathbb{R},
$$

is such that its representative $F$ fulfills the system of second-order PDEs

$$
\frac{\partial^{2} F}{\partial p_{i j} \partial p_{h k}}=0, \quad \forall i, j, h, k .
$$

Equation 108, that is a PDE imposed on the left-hand side of another PDE (in this case, $\mathcal{E})$, is what we shall call a test later on. The key feature of 108 is that it is not $\operatorname{Cont}(M)$-invariant. Making (108) into a Cont $(M)$-invariant test is not an easy task, and the heavy machinery used in [22] confirms that; see also [36]. Nevertheless, the result is surprisingly simple, and even easy to guess. If we declare that $\mathcal{E}$ passes the Monge-Ampère test if and only if

$$
\frac{\partial^{2} F}{\partial p_{(i j} \partial p_{h k)}}=0, \quad \forall i, j, h, k, \quad \text { for some representative } F \text { of } \mathcal{E},
$$

then this test is $\operatorname{Cont}(M)$-invariant. The curious reader may run it on 105 just for fun.

In the paper $[22$ the authors have proved that a (symplectic) second order PDE $\mathcal{E}$ passes the Monge-Ampère test if and only if $\mathcal{E}=\{F=0\}$, with

$$
F=A+B^{i j} p_{i j}+C^{\bullet}(2 \times 2 \text { minors })+\ldots+D^{i j} p_{i j}^{\#}+D \operatorname{det}\left(p_{i j}\right),
$$

which coincides with the classical definition of a (general) Monge-Ampère equation with constant coefficients, see, e.g. [2, Formula (0.5)]. Bearing in mind the definition of Plücker

\footnotetext{
${ }^{9}$ The simplest case of such a function is $f=0$.
} 
coordinates (see $(18), 20)$ and $(21)$ ), it is easy to see the geometry behind formula 110$)$ : it is nothing but the equation of a hyperplane section of $\operatorname{LGr}(n, 2 n)$, namely the intersection of $\operatorname{LGr}(n, 2 n)$ with the hyperplane

$$
\left[A: B^{i j}: C^{\bullet}: \ldots: D^{i j}: D\right] \in \mathbb{P}\left(V_{\lambda_{n}}^{*}\right) .
$$

The correct way to formulate the Monge-Ampère test is via the so-called BGG resolution. The same technique provides a similar test for hypersurface sections of higher degree, that is, with $F$ being a (homogeneous) polynomial of all the minors of $p_{i j}$ of a certain degree $r>1$. Observe that this notion of (algebraic) degree has nothing to do with the order of the PDE, which is always 2. For instance, 105 and $p_{11}^{2}$ are both quadratic in the $p_{i j}$ 's, however the former is linear in the Plücker coordinates, whereas the latter is quadratic. The aforementioned BGG technique is explained in the paper [22].

2.6. Goursat-type Monge-Ampère equations. A similar expression to (105 describes the so-called Goursat-type (resp., symplectic Goursat-type) Monge-Ampère equation

$$
\operatorname{det}\left(p_{i j}-D_{i j}\right)=0
$$

where $D_{i j}$ is a (not necessarily symmetric) $n \times n$ matrix of functions on $M$ (resp., of constants). It is natural to ask oneself whether the class of (symplectic) Goursat-type Monge-Ampère equations is a proper subclass of the class of (symplectic) Monge-Ampère equations. A straightforward count of the parameters immediately says yes. Let us begin with $n=2$. It was already pointed out that the space parametrising the hyperplane sections of $\operatorname{LGr}(2,4)$ - that is, (symplectic) Monge-Ampère equations in two variablesis the dual $\mathbb{P}^{4 *}$ of the Plücker embedding space, see Section 1.7 On the other hand, the space of matrices $D_{i j}$ is also 4-dimensional, so that, topological obstruction aside, the two classes of PDEs may well coincide.

Over the complex field, they indeed do.

Over the reals, we have $\mathcal{E}=\{F=0\}$, with

$$
\begin{aligned}
F=\operatorname{det}\left(p_{i j}-D_{i j}\right) & =\operatorname{det}\left(p_{i j}\right)-D_{22} p_{11}+\left(D_{12}+D_{21}\right) p_{12}-D_{11} p_{22}+\operatorname{det}\left(D_{i j}\right) \\
& =E \operatorname{det}\left(p_{i j}\right)+A p_{11}+2 B p_{12}+C p_{22}+\Delta
\end{aligned}
$$

and, independently on $D$, the equation $\mathcal{E}$ is always non-elliptic since

$$
\left(A C-\Delta E-B^{2}\right)=-\left(\frac{D_{12}-D_{21}}{2}\right)^{2} \leq 0 .
$$

In other words, for $n=2$, the subclass of Goursat-type Monge-Ampère equations coincides with the open subclass of non-elliptic Monge-Ampère equations. For $n=3$ a simple dimension count shows that this is no longer possible: the class of (symplectic) Goursat-type Monge-Ampère equations is $(3 \cdot 3=9)$-dimensional, whereas all (symplectic) Monge-Ampère equations are parametrised by $\mathbb{P}^{13 *}$.

\footnotetext{
${ }^{10}$ Here we have employed the same notation used in [9, p. 588] for the definition of elliptic/parabolic/hyperbolic Monge-Ampère equations in two dimensions - up to the replacement of symbols $\Delta \leftrightarrow D$. Observe that the inequality (114) becomes an equality (that is, $\mathcal{E}$ is parabolic) if and only if the matrix $D$ is symmetric.
} 
From a geometrical standpoint, as we have already stressed in Section 1.16, the equation 112 is nothing but the Lagrangian Chow form of the $(n-1)$-dimensional (linear) variety $\mathbb{P} D$ in $\mathbb{P}\left(V \oplus V^{*}\right)$. Then we are just saying that, in general, not all the hyperplane sections are the Lagrangian Chow transform of a linearly embedded $\mathbb{P}^{n-1}$ inside the projectivised symplectic space.

The reader should be aware of the fact that, in the general context of second order PDEs - i.e., when the hypothesis of being symplectic has been dropped - the $n \times n$ matrix $D$ is allowed to depend on the point of $M$. In other words, the $n$-dimensional subspace $D$ (which we keep denoting by the same symbol $D$ ) is actually an $n$-dimensional subdistribution of the contact distribution on $M$. It is $\operatorname{Cont}(M)$-equivariantly associated to the equation 112 itself. The context will always make it clear, whether $D$ is a distribution or an $n$-dimensional subspace.

Recall that, for $n=2$, the two double fibration pictures 90 and $(91)$ coincide. Then the Lagrangian Chow transform can be "inverted" simply by taking the characteristic lines (which for $n=2$ are the same as hyperplanes). More precisely, given any $\mathcal{E} \subset$ $\operatorname{LGr}(2,4)$, one defines

$$
X_{\mathcal{E}}:=\left\{\Sigma \in \mathbb{P}\left(V \oplus V^{*}\right) \mid \Sigma^{(1)} \text { is tangent to } \mathcal{E} \text { in some point }\right\} .
$$

In other words, $X_{\mathcal{E}}$ is the union of all the characteristics of $\mathcal{E}$ in all its points. More precisely, for any $L \in \mathcal{E}$ consider the characteristic variety $\Xi_{L}(\mathcal{E})$ : the points of the latter are, by definition, hyperplanes in $L$, that is lines in $L$. But $L$ is contained into $V \oplus V^{*}$, so that lines in $L$ are also lines in $V \oplus V^{*}$, that is points of $\mathbb{P}\left(V \oplus V^{*}\right)$. So, definition 115 can be rephrased as

$$
X_{\mathcal{E}}=\bigcup_{L \in \mathcal{E}} \Xi_{L}(\mathcal{E})
$$

We stress that the characteristic variety $\Xi(\mathcal{E})$ is a bundle over $\mathcal{E}$, whereas $X_{\mathcal{E}}$ is a onedimensional sub-distribution of $\mathbb{P}(\mathcal{C})$, that is a 2-dimensional conic sub-distribution of the contact distribution $\mathcal{C}$ [T5, Section 7].

If the equation $\mathcal{E}$ is the Goursat-type Monge-Ampère equation associated, according to 112 to the subdistribution $D \subset \mathcal{C}$, then

$$
X_{\mathcal{E}}=\mathbb{P} D \cup \mathbb{P} D^{\perp},
$$

where $\ldots{ }^{\perp}$ means the symplectic orthogonal.

Observe that the equation 112 above remains unchanged if $D$ is replaced by its orthogonal - the matrix counterpart of taking the symplectic orthogonal. Then it is not an exaggeration to claim that a Goursat-type Monge-Ampère equation $\mathcal{E}$ is unambiguously determined by its "inverse Lagrangian Chow form" $X_{\mathcal{E}}$. Due to the invariance of the framework, the sub-distribution $X_{\mathcal{E}}$ of $\mathcal{C}$ can by all means replace $\mathcal{E}$ in the treatment of the equivalence problem. This point of view is at the basis of many works about invariants and classification of Goursat-type Monge-Ampère equations, see, e.g., [4, 15, 9, 13, 29, 30].

It is worth noticing that the analogous construction of $X_{\mathcal{E}}$ for multidimensional PDEs is slightly more complicated [2]. The class of PDEs "that are the Lagrangian Chow transform of their own inverse Lagrangian Chow transform"-reconstructable, for shortcontains in fact more than the Goursat-type Monge-Ampère equations, but it has not yet been explored completely. 
2.7. Low-dimensional examples. Let us recall that, for $n=2$, the space $\mathbb{P}^{4 *}$ naturally parametrises hyperplane sections of $\operatorname{LGr}(2,4)$, that is (symplectic) two-dimensional Monge-Ampère equations, see Section 1.7 and Section 2.5. Inside $\mathbb{P}^{4 *}$ there sits the threedimensional dual variety $\operatorname{LGr}(2,4)^{*}$, viz.,

$$
\underbrace{\operatorname{LGr}(2,4)^{*}}_{\text {parabolic Monge-Ampère }} \subset \underbrace{\mathbb{P}^{4 *}}_{\text {all Monge-Ampère }},
$$

and it corresponds precisely to the sub-class of parabolic (Goursat-type symplectic) Monge-Ampère equations, see Section 2.6. Indeed, when $D$ is Lagrangian, i.e., symmetric, the symbol of $F$ has a double root, see Section 2.2 and 113 . Since we are working over the reals, between the subset and the whole space there is also the open domain made of non-elliptic Monge-Ampère equations, that is all Goursat-type Monge-Ampère equations 112.

For $n=3$ the stratification becomes more interesting, since the dual variety is singular. We are now in position of interpreting 30 in terms of Monge-Ampère equations:

$$
\underbrace{\operatorname{Sing}\left(\operatorname{LGr}(3,6)^{*}\right)}_{\text {parabolic Monge-Ampère }} \subset \underbrace{\operatorname{LGr}(3,6)^{*}}_{\text {linearisable Monge-Ampère }} \subset \underbrace{\mathbb{P}^{13 *}}_{\text {all Monge-Ampère }} .
$$

The (9-dimensional) domain of Goursat-type Monge-Ampère equations is between the first two strata. A proof of the fact that the 12 -dimensional variety $\operatorname{LGr}(3,6)^{*}$ corresponds to linearisable Monge-Ampère equation can be found in [18, Section 3.6] or in [T4, Theorem 1.4].

The cases $n=3$ and $n=4$ are important in that another class of Monge-Ampère equations, which is trivial for $n=2$, begins to show up. This is the class of integrable Monge-Ampère equations (by the method of hydrodynamic reductions), which will be briefly explained in Section 2.8 below. For $n=3$ these coincide with the linearisable ones. From 119 it follows that there exist non-integrable Monge-Ampère equations. In fact, these are the general ones, since they form two open orbits, represented by

$$
\operatorname{det}\left(p_{i j}\right)=1, \quad \operatorname{det}\left(p_{i j}\right)=\operatorname{tr}\left(p_{i j}\right),
$$

see [18, Equation (13)]. In the same paper it is proved that the space of integrable Monge-Ampère equations has dimension 21. Since linearisable (that is the same as integrable) Monge-Ampère equations correspond to the 12 -dimensional variety $\operatorname{LGr}(3,6)^{*}$, pure dimensional considerations show that there is a lot of integrable second order PDEs that are not of Monge-Ampère type.

The picture begins to change starting from $n=4$. First of all, integrable MongeAmpère equations do not coincide with the linearisable ones, [T4, Theorems 1.4, 1.5, 1.6]. Second, the size of the space parametrising integrable second order PDEs does not grow, as a function of $n$, as fast as the size of the sub-variety of $\operatorname{LGr}(n, 2 n)^{*}$ parametrizing integrable Monge-Ampère equations. This simple observation led Ferapontov and Doubrov to conjecture, in 2010, that from $n \geq 4$ a (symplectic) integrable second order PDE must be necessarily an (integrable) Monge-Ampère one [16, Section 1]. Even though the case $n=4$ has been recently solved by Ferapontov, Kruglikov and Novikov [19], to date the conjecture is still unanswered in general. 
2.8. Integrability by the method of hydrodynamic reductions. As the reader may have noticed, our survey of the geometry of Lagrangian Grassmannians, their hypersurfaces and second order PDEs has begun to border with ongoing research activities and open problems. It is then the appropriate moment to end it. We will just mention a few recent research results and current projects to the benefit of the most curious readers, see Section 2.9 below.

Before that, we briefly outline the notion of hydrodynamic integrability, in view of the central role played here by the Ferapontov conjecture. The special classes of MongeAmpère equations introduced so far -including the integrable ones - can all be interpreted as suitable $\mathrm{Sp}_{2 n}$-invariant subsets in $\mathbb{P} V_{\lambda_{n}}^{*}$. Nevertheless, the notion of hydrodynamic integrability was born originally at the antipodes of Algebraic Geometry in response to a rather tangible problem, which is worth recalling.

The first historically recorded "hydrodynamic reduction" of a three-dimensional quasi-linear system of PDEs dates back to 1860 and it is due to Riemann. His paper [40] provides a mathematical treatment of a problem of nonlinear acoustics proposed by von Helmholtz - the propagation of planar air waves. The system of PDEs describing the problem expressed the temperature $T$ as a function of the three independent variables $\rho, p, v$ - density, pressure and velocity. Riemann's method consisted in postulating the existence of solutions depending on two auxiliary independent variables $r$ and $s$, and then solving the so-obtained reduced system.

In 1996, a similar method was employed by Gibbons and Tsarev in order to obtain a "chain of hydrodynamic reductions" [20] out of a famous multidimensional system of PDEs introduced by Benney in the seventies [7. Unlike Riemann's work, the soobtained chain of hydrodynamic reductions does not lead to actual solutions of the original system of PDEs, but the fact that each reduction is compatible reflects a (still unspecified) property of integrability of the system itself.

The idea that a multidimensional (system of) PDEs may be called "integrable" if the corresponding "hydrodynamic reductions" - obtained from it by a suitable (though straightforward) generalization of the original Riemann's method - are compatible finally reached its maturity in the early 2000's thanks to the works of Ferapontov and his collaborators (see [18 and references therein). They observed that the condition of being integrable (in the sense of hydrodynamic reductions) singles out a nontrivial subclass in the class of second order symplectic PDEs, that is precisely the one mentioned in Section 2.7. They also obtained, for three-dimensional systems, an integrability test, that is a PDE imposed on the left-hand side of an unknown symplectic second order PDE, which is satisfied if and only if the unknown PDE is hydrodynamically integrable. Unlike the aforementioned Monge-Ampère test (109), which is a consequence of the general construction of the BGG resolution ${ }^{11}$ Ferapontov's method was based on computer-algebra computations and this is why the integrability test is now known only for small values

\footnotetext{
${ }^{11}$ Due to obvious limitations, the details of the Monge-Ampère test based on the BGG resolution cannot be reviewed here. In Section 1.13 above we have sketched the idea behind it, but for a full account of it the reader should consult 22 .
} 
of $n$ (to date, only 3 and 4). In terms of these tests, Ferapontov conjecture may be recast as follows:

$$
\text { (Monge-Ampère test) }+ \text { (integrability test) } \equiv \text { (integrability test) } \forall n \geq 4 \text {. }
$$

It then all boils down to formalise 121 in a framework which is rich and general enough to make it possible elaborate an answer. A promising technique is based on the $\mathrm{CO}_{n^{-}}$ structure associated with a non-degenerate hypersurface in $\operatorname{LGr}(n, 2 n)$, see Section 2.9

In order to see what really means for a (symplectic) $\operatorname{PDE} \mathcal{E}=\left\{F\left(p_{i j}\right)=0\right\}$ to be integrable in the aforementioned hydrodynamical sense, we need to explain in detail the notion of a $k$-phase solution of $\mathcal{E}$. Since $\mathcal{E}$ is symplectic, we can identify $\mathcal{E}$ with its fibre, that is a hypersurface in $\operatorname{LGr}(n, 2 n)$. Then $f \in C^{\infty}\left(\mathbb{R}^{n}\right)$ is a solution to $\mathcal{E}$ if its Hessian matrix, understood as a Lagrangian plane parametrised by points of $\mathbb{R}^{n}$, takes values into $\mathcal{E}$ (combine (98) and 102 ). In the streak of the aforementioned Riemann's original work, we understand a $k$-phase solution as a solution $f$ which depends on the independent variables $\left(x^{1}, \ldots, x^{n}\right) \in \mathbb{R}^{n}$ through the auxiliary variables $\left(R^{1}, \ldots, R^{k}\right) \in \mathbb{R}^{k}$, in such a way that the coordinate vector fields $\frac{\partial}{\partial R^{i}}$ have rank one, see Section 1.14 . In terms of commutative diagrams,

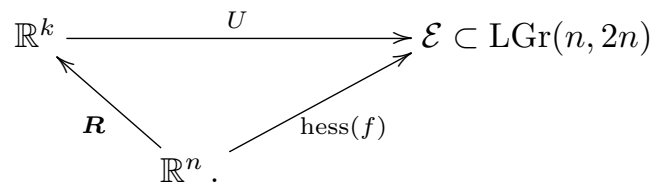

It is no coincidence that these $R^{i}$ 's are called Riemann invariants. A $k$-phase solution of $\mathcal{E}$ is precisely a solution $f$ of $\mathcal{E}$ making commutative the above diagram, with

$$
\operatorname{rank}\left[U_{*}\left(\frac{\partial}{\partial R^{i}}\right)\right]=1, \quad \forall i=1, \ldots, k .
$$

Basically, a PDE $\mathcal{E}$ is declared to be integrable if it possesses "sufficiently many" $k$-phase solutions, for all $k$ (even if it suffices to check it just for $k=2,3$ ). More precisely, one couples the given equation $\mathcal{E}$ with auxiliary equations expressing the existence of the functions $U$ and $\boldsymbol{R}$ and, most importantly, encoding the rank-one condition (123). Then $\mathcal{E}$ is declared to be integrable if the so-obtained system is compatible. More details can be found, e.g., in [18].

It is worth observing that the (physically motivated) notion of a $k$-phase solution corresponds to the purely algebro-geometric concept of a $k$-secant variety. This interesting parallel is the main motivation behind the recent work of Russo [T4].

2.9. A selection of recent research results. The main consequence of the nondegeneracy of a hypersurface $\mathcal{E}$ in $\operatorname{LGr}(n, 2 n)$ is the presence of a $\mathrm{CO}_{n}$-structure on $\mathcal{E}$. This is essentially due to the reduction of $\mathrm{GL}(V)$, the zero-degree part of $P$, to $\mathrm{CO}(\operatorname{Smbl}(\mathcal{E}))$, the subgroup of $\mathrm{GL}(V)$ preserving the line $\operatorname{Smbl}(\mathcal{E})$, see definition (101). Because the $P$-principal bundle $\mathrm{Sp}_{2 n} \longrightarrow \operatorname{LGr}(n, 2 n)$ is made of second-order frames (see Section 1.11, such a $\mathrm{CO}_{n}$-structure on $\mathcal{E}$ is not a conformal metric. This makes things even more intriguing. 
There is not yet in the literature a systematic treatment of such $\mathrm{CO}_{n}$-structures and this is not the appropriate place to start one. Worth to mention however is the skilful work [41] by Smith, where $n$ is assumed to be 3 and hence $\mathrm{CO}_{3}$-structures are the same as $\mathrm{GL}_{2}$-structures. There the author even goes beyond the class of hypersurfaces (5-folds) in $\operatorname{LGr}(3,6)$, and studies the equivalence problem of arbitrary $\mathrm{GL}_{2}$-structures in dimension 5. Invariants are extracted from a preferred principal connection which is associated with each such structure. In particular, he finds the embeddability conditions (i.e., those ensuring that an abstract $\mathrm{GL}_{2}$-structure in dimension 5 can be realised as a hypersurface in $\operatorname{LGr}(3,6))$ and lists several non-equivalent classes of second order symplectic PDEs in three independent variables.

An analogous treatment of the 4-dimensional case is still lacking in the literature. Nevertheless it is worth to mention the recent preprint by Ferapontov, Kruglikov and Novikov, who answered the Ferapontov conjecture for $n=4$ [19].

Concerning the Lagrangian Chow form and the correspondence between substructures of the contact distribution and second order PDEs, it is worth to mention the work [44] by The and the almost simultaneous work [3] by the authors and Alekseevsky. The problem dealt with there is that of constructing a PDE admitting a prescribed simple (complex) Lie group of symmetries. The departing point is the so-called sub-adjoint variety [T5], Section 8] of a rational homogeneous contact manifold, which is an example of a conic subdistribution of the contact distribution, see Section 1.16. Besides these highly symmetric cases, there is still no systematic treatment of "higher degree" analogues of Goursat-type Monge-Ampère equations.

Especially to the reader who is wondering "why always second order" we may suggest the paper [31] where an analogous approach to the one proposed here has been applied to the natural third order analogues of Monge-Ampère equations.

3. Appendix: a guide to reading this volume. The present paper was entirely dedicated to the geometry of the Lagrangian Grassmannian and its hypersurfaces. However, it should not be forgotten that the Lagrangian Grassmannian bundle $M^{(1)}$ over a contact manifold $M$ is but an example of the variety of integral elements of an Exterior Differential System (EDS). The theory of EDS'es represents one of the most general frameworks for studying (system of) PDEs from the point of view of differential geometry (an alternative approach is based on jet spaces [28]). It was born with the pioneering works of Pfaff [38, Frobenius and Darboux [14] and Cartan [12]. Later it was perfected through many contributions. All details about the modern incarnation of theory can be found in the excellent book [8] by Bryant, Chern, Gardner, Goldschmidt and Griffiths. However, the size of the volume may be discouraging for those who seek a swift and workable introduction to the topic. McKay's paper [T2] serves precisely such a purpose.

Smith's paper [T3] is, in a sense, complementary to McKay's one. While the latter is concerned with differential ideals and PDEs, the former focuses instead on the geometry of the set of integral elements of an EDS, understood as a sub-bundle of the Grassmannian bundle. The vertical bundle of these sub-bundles, known as tableaux, the characteristic variety and its incidence correspondence are all examined in detail. 
The paper [T4] by Russo is a natural companion to the works by Ferapontov and his collaborators on the geometry of hydrodynamic integrability [16, 18]. The author carefully explains several algebro-geometric notions and theorems that are made use of, more or less explicitly, in Ferapontov's works. In particular, some classical results on the geometry of secant varieties are reviewed, and a nice technique is employed to deal with the cases of $\operatorname{LGr}(3,6)$ and $\operatorname{LGr}(4,8)$ : the analogy of these cases with the twisted cubic in $\mathbb{P}^{3}$ and the rational normal curve in $\mathbb{P}^{4}$, respectively.

The notion of a contact structure does not pertain exclusively to the realm of differentiable manifolds. The parallel idea of a complex contact manifold is reviewed in [T5], by Buczyński and one of us (Moreno). However, the main purpose of the paper is that of underlying important and unexpected bridges between the complex-analytic and the real-differentiable setting. In particular, there are discussed the twistor correspondence for quaternion-Kähler manifolds and certain substructures of the contact distribution that can be studied via the Cartan's method of equivalence.

Panasyuk's paper [T6] reviews an interesting correspondence, basically due to Gelfand and Zakharevich, between the notion of a bi-Hamiltonian system and the notion of a Veronese web. The former is a powerful tool, widely exploited in the theory of integrable systems, that are PDEs admitting a particularly rich and well-behaved set of (higher) symmetries and/or conservation laws. The latter is a purely geometric construction, generalising that of a web: it is a family, parametrised by $\mathbb{P}^{1}$, of foliations such that the annihilators describe a rational normal curve in the projectivised cotangent bundle. Such a geometric interpretation adds some clarity to the integrable systems' area of research, which features many excellent techniques but sometimes lacks theoretical rigor.

The reader who was surprised by the identification of $\operatorname{LGr}(2,4)$ with the Lie quadric may appreciate Jensen's short review of Lie sphere geometry [T7], which deals with the space $\mathcal{S}\left(\mathbb{R}^{3}\right)$ of generalised spheres in the Euclidean space $\mathbb{R}^{3}$. "Generalised" means that it encompasses the spheres of zero radius (points) and those of infinite radius (planes) as well. Consider the pseudo-Euclidean vector space $V=\mathbb{R}^{4,2}$ of signature $(4,2)$. Then $\mathcal{S}\left(\mathbb{R}^{3}\right)$ is identified with the points of the Lie quadric $Q=\mathbb{P} V_{0}$, that is the projectivisation of the isotropic cone $V_{0}$ in $V$. The author studies the quadric as a homogeneous space $Q=\mathrm{O}(4,2) / P$ where $P$ is the parabolic subgroup that stabilises an isotropic line. Lines in $Q$ correspond to two-dimensional absolutely isotropic 2-planes in $V$.

Finally, Musso and Nicolodi's paper [T8 provides a lucid introduction to Laguerre geometry with a clean presentation of the fundamental constructions. It contains helpful comparisons to surface theory in other, classical geometries. Its subject represents a perfect arena to show the potential of the standard method of moving frames and of EDS'es. The reader will be pleased to see how geometry adds some perspective and helps to demystify the more technical aspects of the Cartan-Kähler theorem as well as of the frame adaptation.

\section{References to papers from this volume}

[T2] B. McKay, Introduction to exterior differential systems, this volume, 45-55.

[T3] A. D. Smith, Involutive tableaux, characteristic varieties, and rank-one varieties in the geometric study of PDEs, this volume, 57-112. 
[T4] F. Russo, Projective duality and non-degenerated symplectic Monge-Ampère equations, this volume, 113-144.

[T5] J. Buczyński, G. Moreno, Complex contact manifolds, varieties of minimal rational tangents, and exterior differential systems, this volume, 145-176.

[T6] A. Panasyuk, Kronecker webs, Nijenhuis operators, and nonlinear PDEs, this volume, 177210.

[T7] G. R. Jensen, Lie sphere geometry in $\mathbb{R}^{3}$, this volume, 211-222.

[T8] E. Musso, L. Nicolodi, Surfaces in Laguerre geometry, this volume, 223-255.

\section{Other references}

[1] D. Alekseevsky, R. Alonso-Blanco, G. Manno, F. Pugliese, Monge-Ampère equations on (para-)Kähler manifolds: from characteristic subspaces to special Lagrangian submanifolds, Acta Appl. Math. 120 (2012), 3-27.

[2] D. V. Alekseevsky, R. Alonso-Blanco, G. Manno, F. Pugliese, Contact geometry of multidimensional Monge-Ampère equations: characteristics, intermediate integrals and solutions, Ann. Inst. Fourier (Grenoble) 62 (2012), 497-524.

[3] D. V. Alekseevsky, J. Gutt, G. Manno, G. Moreno, Lowest degree invariant second-order PDEs over rational homogeneous contact manifolds, Comm. Contemp. Math. 21 (2019), 1750089.

[4] R. Alonso-Blanco, G. Manno, F. Pugliese, Normal forms for Lagrangian distributions on 5-dimensional contact manifolds, Differential Geom. Appl. 27 (2009), 212-229.

[5] J.-C. Álvarez-Paiva, G. Berck, Finsler surfaces with prescribed geodesics, arXiv:1002.0243.

[6] alvarezpaiva, Is the Lie quadric $Q^{3}$ isomorphic to the Lagrangian Grassmannian LG(2,4)? https://mathoverflow.net/q/165717

[7] D. J. Benney, A general theory for interactions between short and long waves, Studies in Appl. Math. 56 (1977), 81-94.

[8] R. L. Bryant, S. S. Chern, R. B. Gardner, H. L. Goldschmidt, P. A. Griffiths, Exterior Differential Systems, Math. Sci. Res. Inst. Publ. 18, Springer, New York, 1991.

[9] R. L. Bryant, P. A. Griffiths, Characteristic cohomology of differential systems. II. Conservation laws for a class of parabolic equations, Duke Math. J. 78 (1995), 531-676.

[10] A. Cannas da Silva, Lectures on Symplectic Geometry, Lecture Notes in Math. 1764, Springer, Berlin, 2001.

[11] A. Čap, J. Slovák, Parabolic Geometries. I. Background and General Theory, Math. Surveys Monogr. 154, Amer. Math. Soc., Providence, RI, 2009.

[12] É. Cartan, Leçons sur les invariants intégraux, A. Hermann, Paris, 1922.

[13] D. Catalano Ferraioli, A. M. Vinogradov, Differential invariants of generic parabolic Monge-Ampère equations, J. Phys. A 45 (2012), 265204.

[14] G. Darboux, Sur le problème de Pfaff, Bulletin des Sciences Mathématiques et Astronomiques 6 (1882), 14-36, http://eudml.org/doc/85135.

[15] A. De Paris, A. M. Vinogradov, Scalar differential invariants of symplectic Monge-Ampère equations, Cent. Eur. J. Math. 9 (2011), 731-751.

[16] B. Doubrov, E. V. Ferapontov, On the integrability of symplectic Monge-Ampère equations, J. Geom. Phys. 60 (2010), 1604-1616.

[17] O. Eshkobilov, G. Manno, G. Moreno, K. Sagerschnig, Contact manifolds, Lagrangian Grassmannians and PDEs, Complex Manifolds 5 (2018), 26-88. 
[18] E. V. Ferapontov, L. Hadjikos, K. R. Khusnutdinova, Integrable equations of the dispersionless Hirota type and hypersurfaces in the Lagrangian Grassmannian, Int. Math. Res. Not. IMRN 2010, 496-535.

[19] E. V. Ferapontov, B. Kruglikov, V. Novikov, Integrability of dispersionless Hirota type equations in $4 D$ and the symplectic Monge-Ampère property, arXiv:1707.08070

[20] J. Gibbons, S. P. Tsarev, Reductions of the Benney equations, Phys. Lett. A 211 (1996), 19-24.

[21] E. Goursat, Sur les équations du second ordre à $n$ variables analogues à l'équation de MongeAmpère, Bull. Soc. Math. France 27 (1899), 1-34.

[22] J. Gutt, G. Manno, G. Moreno, Completely exceptional 2nd order PDEs via conformal geometry and BGG resolution, J. Geom. Phys. 113 (2017), 86-103.

[23] J. Harris, Algebraic Geometry: A First Course, Grad. Texts in Math. 133, Springer, Berlin, 1992.

[24] R. Harvey, H. B. Lawson, Calibrated geometries, Acta Math. 148 (1982), 47-157.

[25] F. R. Harvey, H. B. Lawson, Jr., Split special Lagrangian geometry, in: Metric and Differential Geometry, Progr. Math. 297, Birkhäuser/Springer, Basel, 2012, 43-89.

[26] S. Kobayashi, K. Nomizu, Foundations of Differential Geometry, vol. II, Wiley Classic Lib., Wiley, New York, 1996, https://books.google.pl/books?id=ajN0aD65Ez8C

[27] I. Kolář, P. W. Michor, J. Slovák, Natural Operations in Differential Geometry, Springer, Berlin, 1993.

[28] I. S. Krasil'shchik, V. V. Lychagin, A. M. Vinogradov, Geometry of Jet Spaces and Nonlinear Partial Differential Equations, Adv. Stud. Contemp. Math. 1, Gordon and Breach, New York, 1986.

[29] A. G. Kushner, Classification of Monge-Ampère Equations, in: Differential Equations. Geometry, Symmetries and Integrability, Abel Symp. 5, Springer, Berlin, 2009, 223-256.

[30] A. Kushner, V. Lychagin, V. Rubtsov, Contact Geometry and Non-Linear Differential Equations, Encyclopedia Math. Appl. 101, Cambridge Univ. Press, Cambridge, 2007.

[31] G. Manno, G. Moreno, Meta-symplectic geometry of $3^{\text {rd }}$ order Monge-Ampère equations and their characteristics, SIGMA Symmetry Integrability Geom. Methods Appl. 12 (2016), 032, 35 pp..

[32] E. Miller, B. Sturmfels, Combinatorial Commutative Algebra, Grad. Texts in Math., Springer, New York, 2004, https://books.google.pl/books?id=CqEHpxbKgv8C.

[33] J. W. Milnor, J. D. Stasheff, Characteristic Classes. Annals of Math. Stud. 76, Princeton Univ. Press, 1974, https://books.google.pl/books?id=5zQ9AFk1i4EC

[34] G. Moreno, The geometry of the space of Cauchy data of nonlinear PDEs, Cent. Eur. J. Math. 11 (2013), 1960-1981.

[35] G. Moreno, Why there is a relation among the second-order minors of a symmetric $4 \times 4$ matrix?, http://mathoverflow.net/q/209058.

[36] G. Moreno, An introduction to completely exceptional second order scalar PDEs, in: 50th Seminar "Sophus Lie", Banach Center Publ. 113, Polish Acad. Sci. Inst. Math., Warsaw, 2017, 275-289.

[37] I. G. Petrovsky, Lectures on Partial Differential Equations, Dover Books on Math., Dover Publ., New York, 1992.

[38] J. F. Pfaff, Methodus generalis, aequationes differentiarum partialium, nec non aequationes differentiales vulgares, utrasque primi ordinis, inter quotcunque variabiles, complete integrandi, Abhandlungen der Preussischen Akademie der Wissenschaften, Phys.-Math. Classe, 1818, https://books.google.pl/books?id=pkFvmgEACAAJ 
[39] A. Rekalo, What does the word "symplectic" mean?, https://mathoverflow.net/q/45162

[40] B. Riemann, The propagation of planar air waves of finite amplitude, in: Classic Papers in Shock Compression Science, Springer, New York, 1998, 109-132.

[41] A. D. Smith, Integrable GL(2) geometry and hydrodynamic partial differential equations, Comm. Anal. Geom. 18 (2010), 743-790.

[42] D. E. Smith, A Source Book in Mathematics, 2 vols., Dover Publ., New York, 1959.

[43] D. The, Conformal geometry of surfaces in the Lagrangian Grassmannian and second-order PDE, Proc. Lond. Math. Soc. (3) 104 (2012), 79-122.

[44] D. The, Exceptionally simple PDE, Differential Geom. Appl. 56 (2018), 13-41.

[45] C. Villani, Topics in Optimal Transportation, Grad. Stud. Math. 58, Amer. Math. Soc., Providence, RI, 2003.

[46] L. Vitagliano, Characteristics, bicharacteristics and geometric singularities of solutions of PDEs, Int. J. Geom. Methods Mod. Phys. 11 (2014), 1460039.

[47] H. Weyl, The Classical Groups: Their Invariants and Representations. Princeton Univ. Press, Princeton, NJ, 1997. 